\title{
Acoustic Waveform Inversion for Ocean Turbulence ${ }^{\mathscr{O}}$
}

\author{
AleXANDER Minakov \\ Department of Geosciences, University of Oslo, Oslo, Norway \\ HENK KEERS \\ Department of Earth Sciences, University of Bergen, Bergen, Norway \\ DMITRIY KOLYUKHIN \\ Trofimuk Institute of Petroleum Geology and Geophysics, Siberian Branch of Russian \\ Academy of Sciences, Novosibirsk, Russia \\ HANS CHRISTIAN TENGESDAL ${ }^{\mathrm{a}}$ \\ Department of Earth Sciences, University of Bergen, Bergen, Norway
}

(Manuscript received 20 October 2016, in final form 3 April 2017)

\begin{abstract}
The seismic oceanography method is based on extracting and stacking the low-frequency acoustic energy scattered by the ocean heterogeneity. However, a good understanding on how this acoustic wavefield is affected by physical processes in the ocean is still lacking. In this work an acoustic waveform modeling and inversion method is developed and applied to both synthetic and real data. In the synthetic example, the temperature field is simulated as a homogeneous Gaussian isotropic random field with the Kolmogorov-Obukhov spectrum superimposed on a background stratified ocean structure. The presented full waveform inversion method is based on the ray-Born approximation. The synthetic seismograms computed using the ray-Born scattering method closely match the seismograms produced with a more computationally expensive finite-difference method. The efficient solution to the inverse problem is provided by the multiscale nonlinear inversion approach that is specifically stable with respect to noise. Full waveform inversion tests are performed using both the stationary and time-dependent sound speed models. These tests show that the method provides a reliable reconstruction of both the spatial sound speed variation and the theoretical spectrum due to fully developed turbulence. Finally, the inversion approach is applied to real seismic reflection data to determine the heterogeneous sound speed structure at the west Barents Sea continental margin in the northeast Atlantic. The obtained model illustrates in more detail the processes of diapycnal mixing near the continental slope.
\end{abstract}

\section{Introduction}

Wave propagation through random media has been studied extensively (Chernov 1960; Rytov et al. 1989; Ishimaru 1999; Sato et al. 2012). Specifically, the

Supplemental information related to this paper is available at the Journals Online website: http://dx.doi.org/10.1175/ JPO-D-16-0236.s1.

${ }^{\text {a }}$ Current affiliation: Statoil ASA, Sandsli, Norway.

Corresponding author: Alexander Minakov, alexander.minakov@ geo.uio.no scattering of sound in a fully developed turbulent flow (thought of as a random medium) has been given attention in a number of theoretical and experimental studies (Tatarskii 1961; Monin and Yaglom 1971). Understanding of the ocean turbulence phenomenon and its role in the energy balance is one of the essential problems of the ocean sciences. In physical oceanography, many applications deal with spectral statistical characteristics of the fluid velocity, temperature, and acoustic wavefield (Thorpe and Brubaker 1983; Goodman 1990; Seim 1995; Ross and Lueck 2003; Lavery et al. 2003). These studies were focused on the fine structure of the ocean $\left(10^{-3}\right.$ to $\left.1 \mathrm{~m}\right)$ and utilized acoustic frequencies in the range of $1-1000 \mathrm{kHz}$. A 


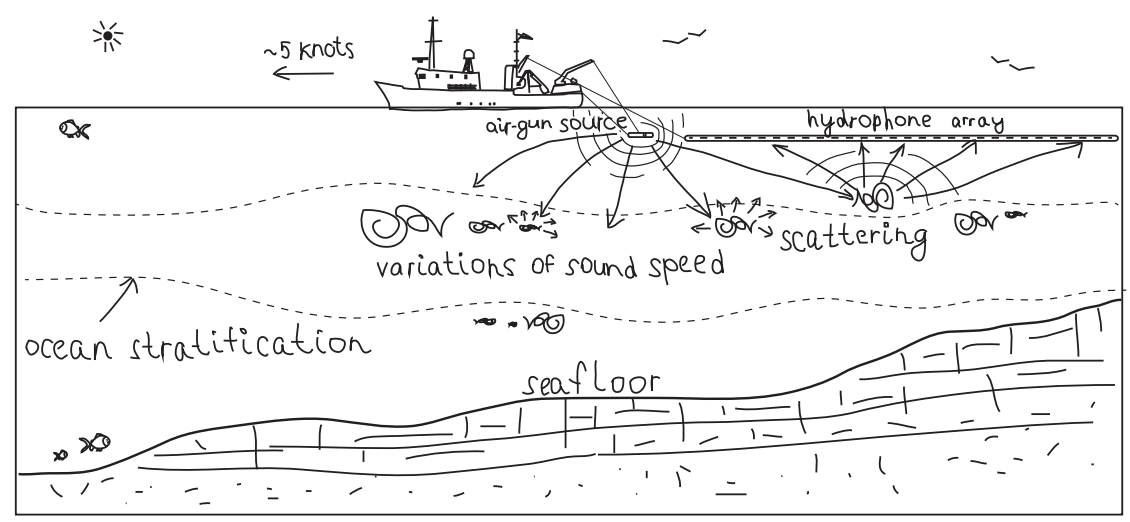

FIG. 1. The concept of seismic oceanography method. The pulses of acoustic signal are produced by a low-frequency pneumatic source (air-gun shots). The signal, scattered on ocean sound speed heterogeneities, is recorded by a few-kilometer-long hydrophone array (streamer). Both source and receivers are towed at shallow depth (about $10 \mathrm{~m}$ ) behind the vessel while it moves at about $5 \mathrm{kt}\left(1 \mathrm{kt}=0.51 \mathrm{~m} \mathrm{~s}^{-1}\right)$.

lower-frequency signal (about $75 \mathrm{~Hz}$ ) has been utilized in the context of long-range horizontal sound propagation and acoustic tomography to investigate the effect of internal waves on acoustics using a forwardscattering approximation (e.g., Colosi et al. 1999).

The method to study the ocean structure using backscattered low-frequency sound $(1-100 \mathrm{~Hz})$ was first demonstrated by Gonella and Michon (1988). Holbrook et al. (2003) brought this method to a wider scientific community. Based on the analysis of the acoustic reflectivity images, this method was found to be useful to study internal waves (Holbrook and Fer 2005), eddies (Biescas et al. 2008), turbulence (Holbrook et al. 2013), and statistical parameters of the ocean flow (Buffett et al. 2010) and is known as seismic oceanography. The concept of this method is schematically shown in Fig. 1.

During the past two decades the seismic full waveform inversion (FWI) became a promising procedure in seismology and seismic exploration (Tarantola 1984; Pratt et al. 1998; Virieux and Operto 2009; Fichtner 2011). Traditional migration methods provide location of the reflectors whereas the FWI recovers the full material parameters (such as sound speed or acoustic impedance) from the data (Bleistein et al. 2013). The FWI of multichannel seismic data has also been applied to predict the 1D temperature-salinity structure of the ocean (Wood et al. 2008; Kormann et al. 2011; Bornstein et al. 2013). An essential part of the FWI consists of solving the forward problem. Kormann et al. (2009) suggested an accurate finite difference (FD) method with special attention to accurate absorbing boundary conditions. However, the low sound speed in water and the artificial reflections put serious computational limitations on the FD methods.
Despite the undoubted usefulness of the seismic oceanography method, there are several other limitations. First, interpretations such as wavenumber spectra characterizing the ocean flow are often made using scaled reflectivity (proportional to the gradient of acoustic impedance) instead of parameter maps (e.g., temperature, sound speed). At the same time, assuming that ocean flow can often be described as a stationary random process, a derivative of this kind of process sometimes cannot be defined (Monin and Yaglom 1971). Second, the ocean is not stationary on the time scale of data acquisition, and this creates additional complications (Vsemirnova et al. 2009). Finally, the simplifying assumption of single scattering underlies most of the migration techniques used to construct reflectivity images.

In the first part of this study, we perform an observing system simulation experiment (OSSE) to address these questions. We analyze the synthetic low-frequency acoustic wavefield perturbed in stochastic turbulent flow. By "low frequency," we mean that the acoustic wavelength $\left(10^{2}-10^{3} \mathrm{~m}\right)$ is larger or comparable to the size of the ocean sound speed fluctuations and much larger than the fluid particle displacement during one source pulse $\left(\approx 10^{-2} \mathrm{~m}\right)$. We suggest an efficient acoustic waveform modeling and inversion technique that makes it possible to go beyond previous 1D approach (Wood et al. 2008; Kormann et al. 2011; Bornstein et al. 2013) and produce 2D/3D models of sound speed. In addition, we describe and use a flexible random field simulation technique and apply it for modeling ocean turbulence in space and time. This technique ensures the recovery of accurate statistical moments over the realizations and thus enables us to 
study a time-averaged sensing of a medium such as in seismic oceanography.

The single-scattering or Born approximation implies that the model and data can be separated into a smooth background sound speed model and a rapidly varying inspace small perturbation of this model. In this case the solution in the reference model can be used to linearize the forward problem (e.g., Coates and Chapman 1990). This approximation is assumed to be valid in the case of seismic oceanography as the sound velocity perturbation is generally not more than a few percent. We show that the accuracy of this method, when applied to a random fluid medium, gives results that are very similar to those obtained using the FD methods. Thus, the perturbation approach avoids computational limitations in previous studies.

This paper is organized as follows. First, we describe a method to create a 3D time-dependent stochastic model of a temperature and sound speed field corresponding to the Kolmogorov-Obukhov spectrum. After that we describe the ray-Born scattering method to compute synthetic seismograms for a given ocean sound speed model. We present synthetic seismograms corresponding to static and time-dependent ocean models. Then we present FWI and imaging results corresponding to these two modeling setups. The quality of the reconstructed models is additionally assessed based on the energy spectrum pattern. We show that the suggested FWI method makes it possible to recover the KolmogorovObukhov's law for both static and time-dependent ocean models. Finally, we apply our waveform inversion method to real seismic reflection data in the vicinity of the western Barents Sea continental margin in the northeast Atlantic.

\section{Stochastic model of turbulence}

The very large ranges of both time $\left(10^{-2}-10^{4} \mathrm{~s}\right)$ and space $\left(10^{3}-10^{-3} \mathrm{~m}\right)$ scales of the turbulent motion put serious limitations on the practical computations of ocean turbulent flows based on direct solution of the NavierStokes equation. Therefore, the numerical solutions are usually difficult to obtain and may involve various types of subgrid-scale parameterization such as used in largeeddy simulation techniques (e.g., Sagaut 2006). In this paper we use another simplified approach utilizing the simulation of 3D time-dependent realizations of temperature random field based on spectral statistical characteristics of ocean turbulence.

Large-scale stochastic simulations of random fields with multiscale resolution (Sabelfeld 1991) have found application in studies of turbulent flow (Kurbanmuradov 1997), flow in porous media (Kolyukhin and Sabelfeld 2005), large-scale density structure of the universe (Sabelfeld 2010), and other problems. In geoscience applications, different simulation techniques are addressed, for instance, in Christakos (2012). The spectral methods based on the fast Fourier transform (FFT) seemingly became the most popular (Holliger et al. 1993; Sato et al. 2012). The method we present here includes a special treatment for the sampling of the wavenumber intervals in order to get physically consistent random realizations with accurate statistics. In addition, our method is more flexible for large simulations than FFT-based methods.

\section{a. Spectral model of turbulent temperature}

In this work we use the randomized spectral model of the high Reynolds number pseudoturbulence described in Kurbanmuradov (1997) and Sabelfeld and Kurbanmuradov (1998). Kurbanmuradov (1997) assumed that the velocity of incompressible turbulent flow can be represented by a Gaussian homogeneous and isotropic random field with the Kolmogorov-Obukhov energy spectrum in the inertial subrange (Monin and Yaglom 1971):

$$
E(k)=\left\{\begin{array}{lll}
C_{1} \bar{\varepsilon}^{2 / 3} k^{-5 / 3} & \text { if } & k \in\left[k_{0}, k_{\text {max }}\right] \\
0 & \text { if } & k \notin\left[k_{0}, k_{\text {max }}\right],
\end{array}\right.
$$

where $C_{1}$ is the universal constant in the KolmogorovObukhov's law $\left(C_{1} \approx 1.4\right)$ and $k=|\mathbf{k}|=\sqrt{k_{1}^{2}+k_{2}^{2}+k_{3}^{2}}$ is the wavenumber. The minimum and maximum wavenumbers are defined as $k_{0}=2 \pi / L_{\max }$ and $k_{\max }=2 \pi / \eta$, respectively; $L_{\max }$ is the external $\left[O\left(10^{2}-10^{3}\right) \mathrm{m}\right]$, and $\eta$ is the internal (millimeter scale) characteristic spatial scale. The lower limit of the inertial subrange is estimated as $\eta=\left(\nu^{3} / \bar{\varepsilon}\right)^{1 / 4}$. Parameter $\bar{\varepsilon}$ is the mean rate of dissipation of kinetic energy (typically $10^{-6}-10^{-10} \mathrm{~W} \mathrm{~kg}^{-1}$ ), and $\nu$ is the kinematic viscosity of the flow (about $10^{-6} \mathrm{~m}^{2} \mathrm{~s}^{-1}$ ). Previously, a similar approach was employed in Kraichnan (1970) to study the diffusion of fluid particles by a random velocity field.

Here, we adopt a similar method for modeling the temperature and sound speed field in the case of turbulent ocean flow. We consider the temperature fluctuations $\delta T(\mathbf{x}, t)$ as a statistically homogeneous random field with spectrum developed from the Kolmogorov similarity hypotheses for high Reynolds (ratio of inertial to viscous forces) and high Péclet (ratio of convective to molecular processes of heat exchange) numbers (Monin and Yaglom 1971):

$$
E_{T}(k)=\left\{\begin{array}{lll}
0.4 C_{T} \bar{N} \bar{\varepsilon}^{1 / 3} k^{-5 / 3} & \text { if } & k \in\left[k_{0}, k_{\max }\right] \\
0 & \text { if } & k \notin\left[k_{0}, k_{\max }\right]
\end{array}\right.
$$


where $C_{T}$ is the universal constant in the temperature spectrum $\left(C_{T} \approx 2.7\right)$ and $\bar{N}$ is the mean rate of dissipation of the turbulent thermal variance $\left(\bar{N} \approx 10^{-6{ }^{\circ}} \mathrm{C}^{2} \mathrm{~s}^{-1}\right)$. Also, we assume that the Prandtl number, which is a dimensionless quantity that characterizes the ratio of viscous diffusion rate to thermal diffusion rate, has an order of unity as it most often happens (Monin and Yaglom 1971).

Following Kurbanmuradov (1997), we assume that the time evolution of the spectrum of the random temperature field can be modeled using an exponential time decorrelation function:

$$
E_{T}(k, t)=E_{T}(k) \exp \left(-\alpha \bar{\varepsilon}^{1 / 3} k^{2 / 3} t\right)
$$

where $\alpha$ is a dimensionless parameter characterizing the temperature decorrelation in time. Using $\alpha=10$ and $\bar{\varepsilon}=10^{-8} \mathrm{~W} \mathrm{~kg}^{-1}$ in Eq. (3), we obtain a half-life time of 470 and $100 \mathrm{~s}$ for the temperature perturbation with a size of 200 and $20 \mathrm{~m}$, respectively.

\section{b. Numerical simulation technique}

An efficient simulation of random fields is an important problem in physical sciences, including geoscience. Most methods are based on the representation of a Gaussian isotropic random field by a stochastic Fourier integral (Monin and Yaglom 1971; Kramer et al. 2007) that can be written in the form

$$
\delta T(\mathbf{x})=\int \sqrt{E_{T}(\mathbf{k})} W(\mathbf{k}) \exp (-i \mathbf{k} \cdot \mathbf{x}) d \mathbf{k},
$$

where $\delta T$ is a random field, $\mathbf{x}$ denotes spatial coordinates, $i=\sqrt{-1}, \mathbf{k}$ is the wavenumber vector, $E_{T}(\mathbf{k})$ is the spectrum of the random field, $W(\mathbf{k})$ is the amplitude spectrum of white noise, and the bold font denotes vectors.

There are various methods that discretize this integral (Kramer et al. 2007). In this contribution we implement the randomization method which estimates a random realization of a stochastic process. We also compute physically constrained wavenumber intervals to produce random fields. The presented method ensures accurate recovery of the mean and correlation function (Kolyukhin and Sabelfeld 2005).

The general technique for simulation of scalar realvalued isotropic homogeneous Gaussian random field with a given spectrum is described in Sabelfeld (1991). We sample $k_{j}$ according to the probability density function $p_{j}(k)$ defined as (Kurbanmuradov 1997):

$$
p_{j}(k)=\left\{\begin{array}{lll}
E_{T}(k) / E_{j} & \text { if } & k \in \Delta_{j} \\
0 & \text { if } & k \notin \Delta_{j}
\end{array}, \quad\right. \text { and }
$$

$$
E_{j}=\int_{\Delta_{j}} E_{T}(k) d k=\frac{1}{n_{0}} \int_{\Delta} E_{T}(k) d k
$$

where

$$
\begin{aligned}
\Delta_{j} & =\left[\tilde{k}_{j}, \tilde{k}_{j+1}\right), \quad J=1 \ldots n_{0} ; \quad \tilde{k}_{1}=k_{0}, \ldots, \tilde{k}_{n_{0}+1}=k_{\max }, \\
\Delta & =\bigcup_{j=1}^{n_{0}} \Delta_{j} .
\end{aligned}
$$

The realizations of temperature random field with the spectrum defined by Eq. (3) and zero mean can be simulated by the formula

$$
\delta T(\mathbf{x}, t)=\sum_{j=1}^{n_{0}} \sqrt{E_{T}\left(k_{j}, t\right)}\left[\zeta_{j} \cos \left(\theta_{j}\right)+\eta_{j} \sin \left(\theta_{j}\right)\right],
$$

where $n_{0}$ is the number of harmonics and $\zeta$ and $\eta$ are independent random variables with zero mean and unit variance.

The phase component in Eq. (8) can be expressed as

$$
\theta_{j}=k_{j} \mathbf{\Omega}_{j} \cdot \mathbf{x}+\tilde{\omega}_{j} t
$$

where $\boldsymbol{\Omega}_{j}$ is an independent three-dimensional random isotropic unit vector. We obtain the ensemble of random realizations by using the expression for the power spectrum Eq. (3) in Eq. (8). We sample the random numbers $\tilde{\omega}_{j}$ and $k_{j}$ by the formulas (Kurbanmuradov 1997)

$$
\begin{aligned}
\tilde{k}_{j+1} & =\left[\left(1-j / n_{0}\right) k_{0}^{-2 / 3}+j / n_{0} k_{\max }^{-2 / 3}\right]^{-3 / 2}, \\
k_{j} & =\left(\tilde{k}_{j}^{-2 / 3}-u_{0}^{2} n_{0}^{-1} C_{1}^{-1} \bar{\varepsilon}^{-2 / 3} \gamma_{1 j}\right)^{-3 / 2}, \text { and } \\
\tilde{\omega}_{j} & =\alpha \bar{\varepsilon}^{1 / 3} k_{j}^{2 / 3} \tan \left[\pi\left(\gamma_{2 j}-1 / 2\right)\right] .
\end{aligned}
$$

Here $\gamma_{1 j}$ and $\gamma_{2 j}$ are mutually independent random numbers uniformly distributed in $[0, \ldots, 1]$, and $u_{0}^{2}=(2 / 3) \int_{\Delta} E(k) d k, j=1, \ldots, n_{0}$. Equations (10)-(12) provide that the wavenumber $k$ is sampled according to the probability density function described by Eqs. (5)(7). Note that $k_{j}$ in the equation is linked to the flow velocity spectrum. It is also important to mention that the presented method is well suited for modeling vector random fields such as turbulent flow velocity. In this case, $\zeta$ and $\eta$ in Eq. (8) will be random vectors.

We assume that the temperature perturbation is linearly related to the sound speed perturbation. For the temperature to sound speed conversion, we use a coefficient derived from the Mackenzie (1981) equation, ignoring second-order terms and pressure dependence:

$$
\delta c(\mathbf{x}) \approx 4.6 \delta T(\mathbf{x}) .
$$




\section{Forward modeling of acoustic wavefield}

In a general moving fluid, the acoustic wavefield must be affected by both heterogeneities and flow velocity. However, in most seismic oceanography applications, the contribution of flow velocities on wave propagation should be small. The phase shift due to ocean currents can be estimated from (Ostashev and Wilson 2015)

$$
\Delta \Phi \approx-2 \pi f \bar{R} \bar{u}_{R} / c^{2},
$$

where $f$ is the frequency of the sound wave, $\bar{R}$ is the distance from the source to the receiver, $\bar{u}_{R}$ is the ocean flow velocity, and $c$ is sound speed. Substituting $20 \mathrm{~Hz}$ for frequency, $3 \mathrm{~km}$ for the propagation distance, $0.3 \mathrm{~m} \mathrm{~s}^{-1}$ for flow velocity, and $1500 \mathrm{~m} \mathrm{~s}^{-1}$ for sound speed, we obtain a phase delay of about $0.05 \mathrm{rad}$ or $\pi / 62$ that can be neglected here. This effect may become important in the case of high frequencies and strong currents.

In this paper we simulate the wave propagation in the turbulent sound speed model by linear acoustic equation

$$
-c^{-2}(\mathbf{x}) \partial_{t}^{2} u(\mathbf{x}, t)+\nabla^{2} u(\mathbf{x}, t)=s(t) \delta\left(\mathbf{x}-\mathbf{x}_{s}\right),
$$

where $u(\mathbf{x}, t)$ is the acoustic pressure that depends on the position (x) and time $(t), c(\mathbf{x})$ is the sound speed, $s(t)$ is the density of the point source at $\mathbf{x}_{s}$, and $\delta$ is the Dirac delta function (DeSanto 1992). Using that, the following assumptions are made: (i) the flow velocities are small when compared to the sound speed and (ii) the heterogeneous density has a small effect on wave propagation. The turbulence model sound speed heterogeneities are assumed to be "frozen" during the propagation time of the acoustic pulse. For a more general treatment of sound propagation in moving heterogeneous media, we refer to Ostashev and Wilson (2015).

We solve Eq. (15) using two methods. The first method is a time-domain FD method. We use an explicit scheme with fourth-order accuracy in space and secondorder accuracy in time. The absorbing boundary conditions are implemented using a sponge method (Cerjan et al. 1985). The second method is based on the ray-Born approximation (Coates and Chapman 1990; Červený 2005) and explained below.

\section{Ray-Born scattering method}

The ray-Born approximation with application to forward and inverse seismic modeling has been used and discussed in more detail by Dahlen et al. (2000), Thierry et al. (1999), Lambaré et al. (2003), Operto et al. (2003), Moser (2012), and others. Here, we present just a general outline of the theory and present our modeling technique.
It is convenient to transform Eq. (15) to the frequency domain. We adopt the following definition for the Fourier transform pair:

$$
\begin{aligned}
f(\omega) & =\int F(t) \exp (i \omega t) d t, \quad \text { and } \\
F(t) & =\frac{1}{2 \pi} \int f(\omega) \exp (-i \omega t) d \omega,
\end{aligned}
$$

where $\omega$ is the angular frequency. The wave equation [Eq. (15)] in the frequency domain becomes the Helmholtz equation:

$$
\omega^{2} c^{-2}(\mathbf{x}) u(\mathbf{x}, \omega)+\nabla^{2} u(\mathbf{x}, \omega)=s\left(\mathbf{x}, \mathbf{x}_{s}, \omega\right) .
$$

In seismic reflection imaging (Tarantola 1984), it is assumed that the pressure wavefield can be approximated by a sum of incident $u_{0}(\mathbf{x}, \omega)$ (direct wave) and scattered pressure wavefield $\delta u(\mathbf{x}, \omega)$ (Bleistein et al. 2013). Similarly, the sound speed model is represented by a smooth background $c_{0}(\mathbf{x})$ and a weak perturbation $\delta c(\mathbf{x})$ :

$$
c(\mathbf{x})=c_{0}(\mathbf{x})+\delta c(\mathbf{x}) .
$$

This gives rise to the first-order Born approximation as an integral over the scattering volume (Clayton and Stolt 1981; Hudson and Heritage 1981; Rytov et al. 1989; Coates and Chapman 1990; Červený 2005; Symes 2008):

$$
\begin{aligned}
& \delta u\left(\mathbf{x}_{s}, \mathbf{x}_{r}, \omega\right) \\
& \quad=2 \omega^{2} s(\omega) \int_{V} G_{0}\left(\mathbf{x}, \omega, \mathbf{x}_{s}\right) \delta c(\mathbf{x}) c_{0}^{-3}(\mathbf{x}) G_{0}\left(\mathbf{x}, \omega, \mathbf{x}_{r}\right) d \mathbf{x},
\end{aligned}
$$

where $\mathbf{x}_{r}$ is the receiver location and $G_{0}\left(\mathbf{x}, \omega, \mathbf{x}_{s}\right)$ and $G_{0}\left(\mathbf{x}, \omega, \mathbf{x}_{r}\right)$ are the source and the receiver Green's function in the background medium, respectively.

The Green's function $G_{0}\left(\mathbf{x}, \omega, \mathbf{x}_{s}\right)$ in $3 \mathrm{D}$ is

$$
G_{0}\left(\mathbf{x}, \omega, \mathbf{x}_{s}\right)=R_{s x}^{-1} \exp \left[i \omega T\left(\mathbf{x}, \mathbf{x}_{s}\right)\right],
$$

where $R_{s x}$ is the source to scatterer geometrical spreading (which is the inverse of the distance in homogeneous background media) and $T$ is the travel time from $\mathbf{x}$ to $\mathbf{x}_{s}$ (e.g., Červený 2005).

For the purpose of 2D synthetic modeling, it is useful to derive the corresponding solution. A 2D Green's function for a smooth acoustic medium has the form

$$
G_{0}\left(\mathbf{x}, \omega, \mathbf{x}_{s}\right)=-(i / 4) H_{0}^{(2)}\left[\omega T\left(\mathbf{x}, \mathbf{x}_{s}\right)\right],
$$

where $H_{0}^{(2)}$ is the zeroth-order Hankel function of the second kind. Using asymptotic expression for the Hankel function (DeSanto 1992), the asymptotic form of 
Green's function [Eq. (22)] for large $|\mathbf{x}|$ is approximated by

$$
G_{0}\left(\mathbf{x}, \omega, \mathbf{x}_{s}\right)=-\frac{i}{4} \sqrt{\frac{2 c_{0}(\mathbf{x})}{\pi \omega R_{s x}}} \exp \left[i \omega T\left(\mathbf{x}, \mathbf{x}_{s}\right)+i \pi / 4\right] .
$$

The receiver Green's functions can be obtained by analogy.

If the background model is heterogeneous, we find the geometrical spreading $R$ and travel time $T$ by ray tracing. Ray paths for the acoustic waves emanating from a source $\mathbf{x}_{s}$ are found by solving the kinematic ray equations

$$
\begin{aligned}
& \frac{d \mathbf{x}}{d t}=c_{0}^{2}(\mathbf{x}) \mathbf{p}(t), \quad \text { and } \\
& \frac{d \mathbf{p}}{d t}=-\frac{1}{c_{0}(\mathbf{x})(t)} \nabla c_{0}[\mathbf{x}(t)],
\end{aligned}
$$

with initial condition $\mathbf{x}(0)=\mathbf{x}_{s}$ and $\mathbf{p}(0)=\mathbf{p}_{0}$ as the initial takeoff direction. The independent parameter is the travel time $t$ along the ray and $\mathbf{p}$ is the slowness vector. We assume that this path always exists and that it is unique. There is therefore no multipathing and the Maslov index is zero (Červený 2005).

The geometrical spreading $R$ is computed by solving the dynamic ray tracing equations:

$$
\begin{aligned}
& \frac{d}{d t}\left(\frac{\partial \mathbf{x}}{\partial q_{i}}\right)=2 c\left(\frac{\partial c_{0}}{\partial \mathbf{x}}\right)\left(\frac{\partial \mathbf{x}}{\partial q_{i}}\right) \mathbf{p}+c_{0}^{2} \frac{\partial \mathbf{p}}{\partial q_{i}}, \text { and } \\
& \frac{d}{d t}\left(\frac{\partial \mathbf{p}}{\partial q_{i}}\right)=c_{0}^{-2}\left(\frac{\partial c_{0}}{\partial \mathbf{x}} \frac{\partial \mathbf{x}}{\partial q_{i}}\right)-c_{0}^{-1} \frac{\partial^{2} c_{0}}{\partial \mathbf{x} \partial \mathbf{x}} \frac{\partial \mathbf{x}}{\partial q_{i}},
\end{aligned}
$$

where $q_{i}(i=1,2)$ are the takeoff angles. The geometrical spreading $R$ is now given by

$$
R=\frac{\partial \mathbf{x}\left(t, q_{1}, q_{2}\right)}{\partial\left(t, q_{1}, q_{2}\right)} .
$$

Thus, we can write the kernel of the integral Eq. (20) as

$$
K\left(\mathbf{x}_{s}, \mathbf{x}, \mathbf{x}_{r}, \omega\right)=2 \omega^{2} s(\omega) G_{0}\left(\mathbf{x}, \omega, \mathbf{x}_{s}\right) G_{0}\left(\mathbf{x}, \omega, \mathbf{x}_{r}\right) c_{0}^{-3}(\mathbf{x}) .
$$

It depends on the source and receiver locations, scattering point, and frequency. A more general formulation of the ray-Born method including reflections at the interfaces can be found in Dahlen et al. (2000).

Equations (20) and (29) in matrix form are

$$
\left(\begin{array}{c}
\delta u_{1} \\
\ldots \\
\delta u_{M}
\end{array}\right)=\left(\begin{array}{c}
K_{11} \ldots K_{1 N} \\
\ldots \\
K_{M 1} \ldots K_{M N}
\end{array}\right)\left(\begin{array}{c}
\delta c_{1} \\
\ldots \\
\delta c_{N}
\end{array}\right) .
$$

Here, $K_{i j}$ represents a single-frequency kernel for the source-receiver pair $i$ and the scattering volume $j$. The seismograms for the scattered wavefield are obtained after repeating the matrix multiplication up to the highest cutoff frequency and taking the inverse Fourier transform for each seismogram (source-receiver pair).

The numerical implementation of ray tracing is done using a fourth-order Runge-Kutta method. We interpolate travel times and amplitudes along the ray paths into the grid nodes $\mathbf{x}$. The interpolation is performed using Delaunay triangulation. This process is repeated for each source and receiver in the model. The integral in Eq. (20) is replaced by summation over all scattering volumes. The single scatterer is approximated by a block with volume $d \mathbf{x}$.

\section{Waveform inversion method}

In this section, we discuss the inverse problem: how can one reconstruct the sound speed $\delta c$ given acoustic data scattered by ocean heterogeneities? We formulate the acoustic waveform inversion as a nonlinear least squares problem. Suppose we have a collection of seismic traces $d$, given at a discrete number of sources $s$, receivers $r$, and frequencies $\omega$. A least squares estimate for $\delta c$ is obtained by minimizing the functional

$$
\begin{aligned}
\chi(\delta c) & =\sum_{s, r, \omega}[d(s, r, \omega)-\delta u(s, r, \omega)]^{2} \\
& =\sum_{s, r, \omega}[d(s, r, \omega)-K(s, r, \omega) \delta c]^{2} .
\end{aligned}
$$

Here, the summation is over all sources, receivers, and frequencies. Let $\mathbf{m}$ be a vector of $\delta c$ estimates. We search for $\mathbf{m}$ which minimizes the misfit functional $\chi(\delta c)$ using a variant of regularized Newton's optimization (Aster et al. 2011; Fichtner 2011):

$$
\mathbf{m}_{n+1}=\mathbf{m}_{n}+\Delta \mathbf{m}_{n},
$$

where the descent direction $\Delta \mathbf{m}$ at iteration $n$ is found by solving the least squares problem linearized by the Born approximation:

$$
\min \left\|\left(\begin{array}{c}
\mathbf{K} \\
\beta^{2} \mathbf{I}
\end{array}\right) \Delta \mathbf{m}_{n}-\left(\begin{array}{c}
\mathbf{d}-\mathbf{u}_{n} \\
0
\end{array}\right)\right\|^{2} .
$$

where $\mathbf{K}$ is the waveform sensitivity kernel [Eq. (29)] written as a matrix, $\mathbf{u}_{n}$ is the ray-Born synthetic wavefield computed at iteration $n, \mathbf{d}$ is the data vector, and $\beta$ is the damping coefficient that determines the relative importance of the data misfit and $\left(L_{2}\right)$ solution norm. This regularization results in a spatially smoothed solution.

We solve the underdetermined problem Eq. (33) using the iterative LSQR method (Paige and Saunders 
1982). We choose the regularization parameter $\beta$ that gives the lowest data misfit. Solving the problem in Eq. (33), we obtain the descent direction $\Delta \mathbf{m}_{n}$. We select a frequency range of the data perturbation $d(s, r, \omega)-\delta u(s, r, \omega)$ and sensitivity kernel $K(s, r, \omega)$ at iteration $n$. The travel times and geometrical spreading at the scattering points are computed by ray tracing in the background model [Eqs. (24)-(28)] and used for all iterations in the sensitivity matrix and to obtain data residuals. At each iteration we use Eq. (20) to produce synthetic data and then transform it to the time domain to obtain data residuals. This significantly reduces the computation costs of forward modeling. Starting with the lowest frequency, we sequentially include higher frequencies into the minimization process. This multiscale approach helps to guide the iterative inversion toward the global minimum as explained in Fichtner (2011).

We assume that the background sound speed model is smooth enough to avoid multipathing in the ray tracing. This is reasonable, as in our case the background model is $1 \mathrm{D}$ with only a slight variation in sound speed. If multipathing takes place in the background model, it still can be possible to apply our inversion algorithm, but the forward problem has to be solved by an extension of ray-Born to include multipathing (which may be called beam-Born) or by a completely numerical method (e.g., finite differences). An example of this approach when applied to seismic data is shown by Tengesdal et al. (2014). It is also possible to include additional acoustic phases (such as multiple reflections) by a linear combination of corresponding sensitivity kernels (Dahlen et al. 2000).

It can be shown that setting the derivative of the objective function to zero, $\nabla_{c} \chi(\delta c)=0$, and ignoring second-order terms results in the normal equation (Tarantola 1984; Symes 2008)

$$
\mathbf{K}^{\dagger} \mathbf{K} \Delta \mathbf{m}_{n}=\mathbf{K}^{\dagger} \mathbf{d}
$$

where $\mathbf{K}^{\dagger}$ is the adjoint of $\mathbf{K}$.

According to the classical imaging condition (Claerbout 1971), the reflector location $\Delta \mathbf{m}^{*}$ can be obtained by correlation of forward-propagated wavefield from the source location and backward-propagated wavefield from the receiver location. Using Eq. (29) and under some general conditions, this leads to

$$
\Delta \mathbf{m}^{*}=\lambda \mathbf{K}^{\dagger} \mathbf{d}
$$

where $\lambda$ is a scaling coefficient. It is possible to see that Eq. (35) is equivalent to Eq. (34). The square matrix $\mathbf{K}^{\dagger} \mathbf{K}$ is the approximate Hessian, which contains scaling factors that correct for illumination and geometrical

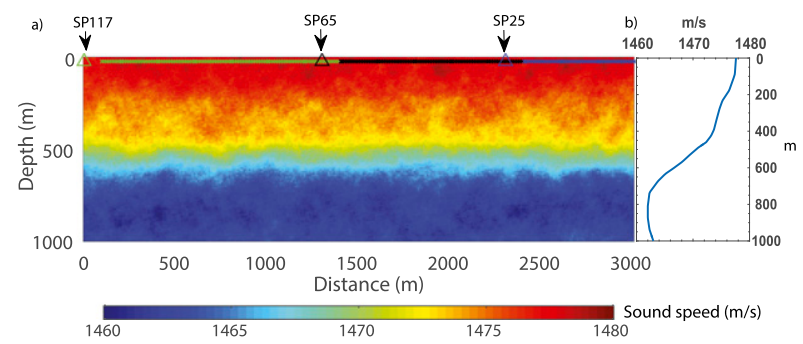

FIG. 2. Synthetic sound speed model. (a) The model is composed of the random sound speed field due to the turbulence model superimposed on a laterally homogeneous background sound speed model. The shot location is shown by triangles and the receiver array (streamer) is shown by dots. The receivers outside the model are not used. Therefore, the number of receivers per shot decreases with the shot point number (SP). The left side of the model is poorly covered by rays since the receivers are located to the right of the source point. (b) Background sound speed profile.

spreading. To find the scaling coefficients, we further approximate the Hessian matrix by its diagonal terms and invert it to find $\lambda$. In the next section, we will use Eqs. (32) and (33) to reconstruct the sound speed variations associated with the turbulence temperature model [Eq. (8)]. In addition, we will use the imaging equation [Eq. (35)] to recover the reflectivity.

\section{Results of synthetic modeling}

\section{a. Parameters of the temperature and sound speed model}

The sound speed model shown in Fig. 2 consists of a 1D layered model on which the perturbation due to the isotropic turbulence is superimposed. The background model is representative for the North Atlantic region as determined by hydrographic transects described in Walczowski (2014). This is a standard sound speed profile characterized by the main thermocline at a depth of 200$1000 \mathrm{~m}$ overlain by a near-surface mixed layer (Ewing and Worzel 1948; Jensen et al. 2011). The model size is $3000 \mathrm{~m} \times 1000 \mathrm{~m}$. The grid spacing is $10 \mathrm{~m}$. We use the parameters to describe the turbulence model as provided by Goodman (1990). The kinematic viscosity $\nu$ is $10^{-7} \mathrm{~m}^{2} \mathrm{~s}^{-1}$ and the average kinetic energy dissipation is $\bar{\varepsilon}=10^{-8} \mathrm{~W} \mathrm{~kg}^{-1}$. The maximum and minimum wavenumbers that describe the turbulent flow in the model are $1.1 \times 10^{4}$ and $0.013 \mathrm{cpm}$, respectively. The Reynolds number is written as $\operatorname{Re}=\left(k_{\max } / k_{0}\right)^{4 / 3}$, so that in our simulation $\mathrm{Re}=0.85 \times 10^{8}$. The mean rate of dissipation of the turbulent thermal variance $\bar{N}=10^{-6}{ }^{\circ} \mathrm{C}^{2} \mathrm{~s}^{-1}$. Klymak and Moum (2007) reported on the measurements of temperature spectra and showed that the turbulence subrange of isopycnal slope spectra can extend to horizontal wavelengths of hundreds of meters. Based on 

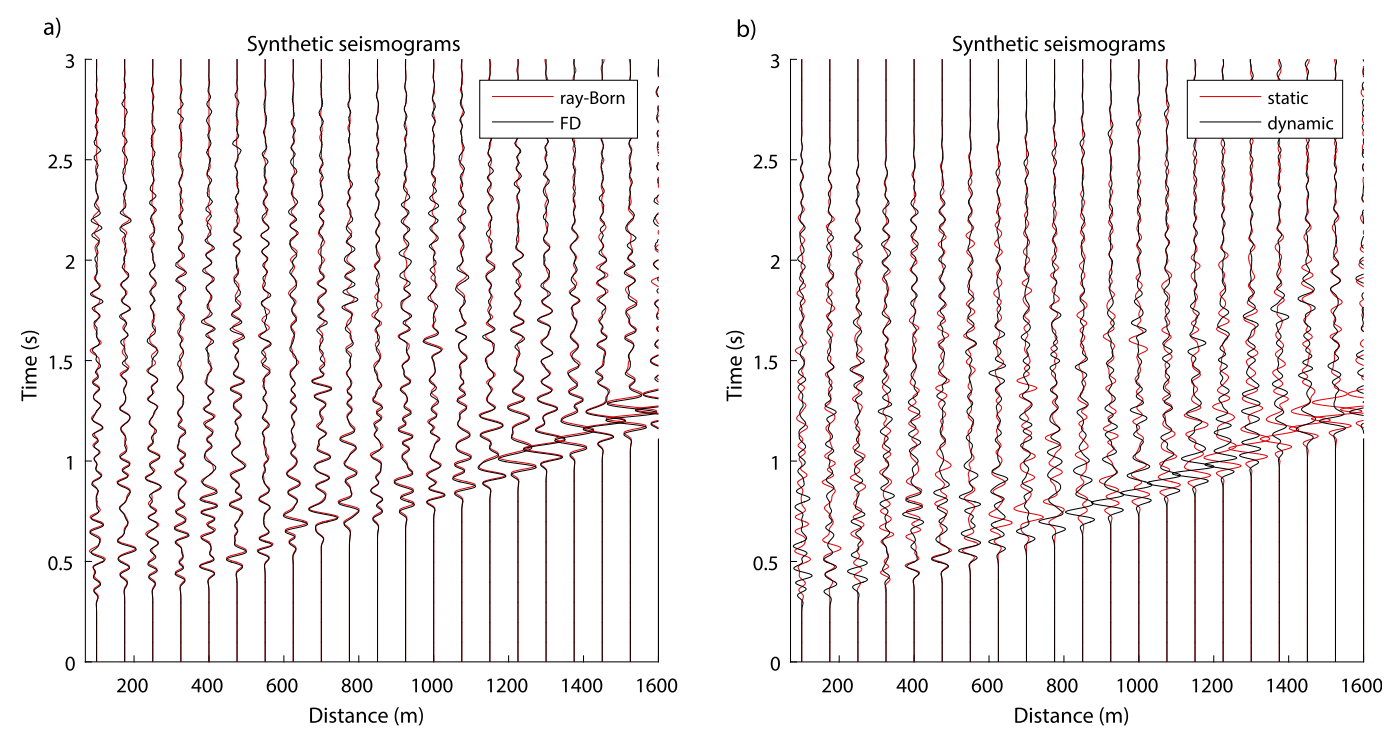

FIG. 3. Synthetic seismograms (SP65). (a) Finite-difference (black) and ray-Born scattering pressure waveforms (red). The direct wave has been removed. (b) Acoustic waveforms corresponding to the static (red) and dynamic sound speed model (black).

their observations, we assume $L_{\max }=500 \mathrm{~m}$. The dimensionless parameter $\alpha$ characterizing the temperature decorrelation in time is chosen to be $\alpha=10$. We use $n_{0}=2000$ harmonics to produce random realizations. We convert temperature fluctuations to sound speed with Eq. (13).

\section{b. Synthetic seismograms}

In this section we present synthetic seismograms computed using the ocean sound speed model described above. The method presented in section 3 is formulated for 3D geometry. However, it is applied to seismic oceanography data, which are usually acquired in along transects. We consider relatively short-range propagation (1-3-km scale) and deep water.

We compute synthetic waveforms using both the rayBorn scattering method and the FD method. The gridcell size for the simulation of wave propagation is $10 \mathrm{~m}$. We use a Ricker wavelet with the peak frequency of $10 \mathrm{~Hz}$ to produce seismograms. The data are presented for a single shot (SP 65 in Fig. 2). The maximum sourcereceiver distance is $2500 \mathrm{~m}$, whereas the receivers are separated by a $12.5-\mathrm{m}$ interval.

In Fig. 3a we present synthetic seismograms for the first realization of the turbulence model (shown in Fig. 2). To compare the 2D FD with the ray-Born solution, we use Green's function of Eq. (23) in Eq. (20). The FD amplitudes were normalized to match the maximum amplitude found using the dynamic ray tracing. We use a single scaling coefficient for all traces. The resulting seismograms computed using the two methods match well. There is no apparent phase misfit. The relative
RMS amplitude misfit is about $3 \%$. Most of the acoustic energy is observed within 1-1.5 s after the arrival time of the direct wave. The close fit of the FD and ray-Born solutions within this time interval indicates that the multiple scattering has a small effect on the acoustic wavefield in our model. However, the effect of multiple scattering can be important for weak later arrivals. This also suggests that the ray-Born scattering method can be used for full waveform modeling and inversion.

The ocean flow velocity is small when compared to the sound velocity. However, during the period of data collection over the same scattering volume $\left(10^{2}-10^{3} \mathrm{~s}\right)$, the ocean structure produced by internal waves and turbulence may change significantly (Vsemirnova et al. 2009). In the Eulerian reference frame, a scattering point would be characterized by the changing with time the scattering radiation strength. To show how this can affect the acoustic wavefield, in Fig. 3b we present synthetic seismograms computed for the moving ocean model. We update the turbulence model every $12.5 \mathrm{~s}$ using the spatial-temporal spectrum of Eq. (3). The modeling geometry is the same as in the previous example. Thus, the difference in the recorded wavefield reflects the change of the scattering strength during the period of data acquisition (120 shots or about $1500 \mathrm{~s}$ in the presented model). In Fig. 3b we observe both amplitude and phase misfit accumulated during 65 shots.

\section{c. Full waveform inversion results}

For the synthetic modeling we use 2D FD acoustic waveform data that we invert with the $2 \mathrm{D}$ ray-Born FWI 

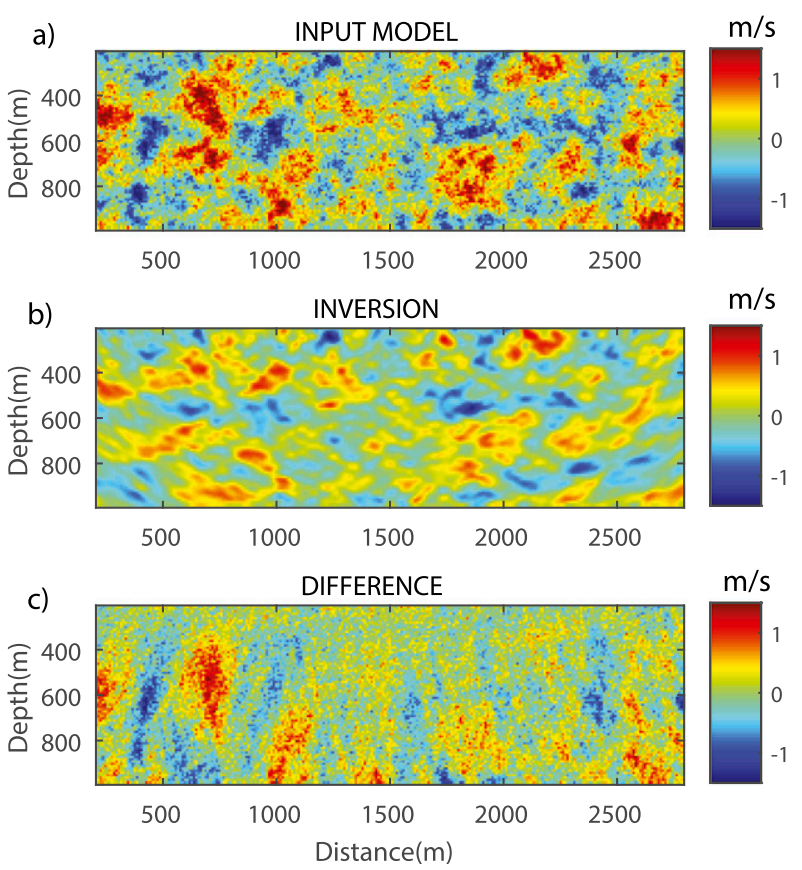

FIG. 4. Full waveform inversion results. (a) Input (true) sound speed perturbation model. Background model is not shown in the figure. (b) Inverted model using the full waveform inversion method. (c) Difference between the true and recovered sound speed perturbations. The model is best recovered in the upper central region. The largest difference is located at the sides (especially at the left side) and the lower boundary of the model where the ray coverage is poor (cf. with the acquisition geometry in Fig. 2).

method. To generate synthetic data we use the acquisition geometry similar to what is employed during acquisition of multichannel seismic reflection data. We produce acoustic waveforms with a $10-\mathrm{Hz}$ Ricker wavelet. The gridcell size for simulations is $10 \mathrm{~m}$. Absorbing boundary conditions are applied at the boundaries to avoid artificial reflections. The interval for sources is $25 \mathrm{~m}$, and receivers are located with a $12.5-\mathrm{m}$ interval. We use an array of receivers with the maximum length of $3000 \mathrm{~m}$ moving to the left in Fig. 2. The active length of the receiver array is limited by the model width. Therefore, the number of receivers per shot reduces from the left to right in Fig. 2. The sound speed model is fixed during the shot time. The model for inversion is parameterized with block scatterers of $10 \times$ $10 \mathrm{~m}$ size (Fig. 4). Since both the seismic data and background sound speed model (generally not a 1D model) are normally defined in space coordinates, we have chosen a pixel parameterization. However, representation of the sound speed model in terms of Fourier series might have had some advantages (e.g., the number of harmonics is substantially smaller than the number of grid points). The sound speed perturbation varies from about -1.5 to $1.5 \mathrm{~m} \mathrm{~s}^{-1}$ in accordance with the turbulence temperature model. The background model is not shown to make the sound speed variation visible. In our model, the upper $200 \mathrm{~m}$ represents the oceanic mixed layer where the temperature is assumed to be nearly uniform, and therefore, the sound speed is fixed. In real data applications, it will be important to include coincident oceanographic data to constrain the model in the vicinity of sources and receivers.

We implement numerically the FWI algorithm using Eqs. (32) and (33). The multiscale frequency-domain inversion approach is similar to that by Pratt et al. (1998). The inversion of the acoustic wavefield consists of a number of iterations. At each iteration, we solve the problem [Eq. (33)] for a single frequency. Similarly, we proceed from the low to high frequencies in such a way that we update the initial model, produce synthetic data, and use the data residual for the next iteration. In the presented examples, the frequency range is between 2 and $20 \mathrm{~Hz}$. We find the data residuals subtract (in the time domain) the ray-Born synthetics corresponding to the inverted sound speed model at given iteration (frequency) from the initial FD data. We obtain the components of the sensitivity matrix Eq. (29) using the background sound speed model. We make use of the amplitude and phase components of Green's solution in Eq. (23), found using the dynamic ray tracing, throughout the inversion process. The oceanographic observations suggest that, on a scale of several kilometers, the background (reference) structure can be accurately modeled using a 1D sound speed distribution with depth. The knowledge of the background model significantly improves the inversion results, but the lack of this information is not a serious limitation (as shown in supplemental material).

\section{1) STATIONARY MODEL OF SOUND SPEED}

The sound speed model, shown in Figs. 2 and 4a, was used to produce synthetic data. This realization corresponds to the last time step of the stochastic turbulence model. In the inverted model (Fig. 4b), both the locations and the shape of the anomalies are well recovered. The magnitude of the anomalies gets slightly smeared with depth because of the natural resolution of the data acquired at the surface. This can also be seen in the difference plot (Fig. 4c). The features with a size of $50 \mathrm{~m}$ or more are well resolved. Smaller-scale structures are not well recovered (especially toward the lower and side boundaries of the model).

The initial misfit is reduced more than 4 times after about 40 iterations when the background model is known exactly. The total misfit reduction is about 2 times smaller in the case when we know only the mean 


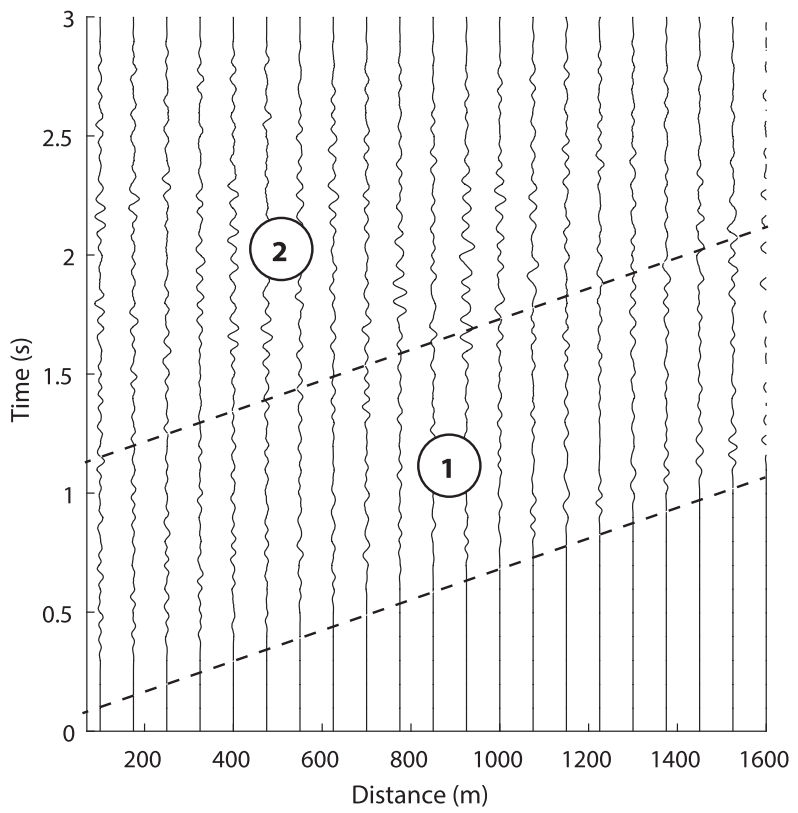

FIG. 5. The misfit between reconstructed and input finite-difference waveforms (shot point SP65) corresponding to the sound speed model in Fig. 4b. Region 1 is where the input and reconstructed waveforms match well. Region 2 is where the final misfit increases because of a combination of limited model resolution and uncertainties related to forward modeling.

value of the sound speed (see supplemental material for additional tests with uniform reference model and different source-receiver geometry). The model in Fig. 4b reproduces data to the modeling accuracy [the total misfit defined by Eq. (33)]. To additionally assess the distribution of the data fit, we plot the difference between the reconstructed and input FD waveforms (Fig. 5). The waveforms match well within about $1 \mathrm{~s}$ after the arrival time of the direct wave. The final misfit increases at a later time. This effect is probably caused by a combination of uncertainties of forward modeling (such as multiple scattering, side reflections, and others) and a limited model resolution at the bottom and toward the sides of the model.

We estimate the mean energy spectrum in the vertical (depth) direction using the recovered sound speed model and reflectivity images. These types of spectral plots should help to recognize small-scale perturbations in the oceans and distinguish them from noise in the data. The energy spectrum is estimated using an averaged vertical periodogram. Apart from the region of high wavenumbers, the spectrum for the inversion matches the spectrum estimated using the input model (Fig. 6). The theoretical turbulence spectrum in the inertial subrange $(-5 / 3$ law) is well recovered. The recovered spectrum is lower than the

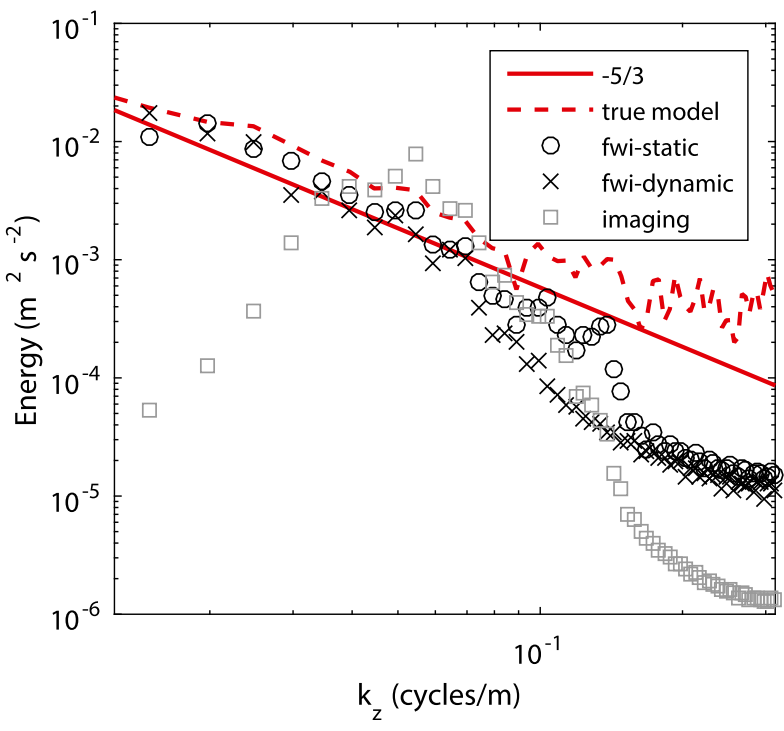

FIG. 6. Sound speed energy spectrum estimated in the depth direction. Shown are the Kolmogorov-Obukhov spectrum (solid line), true input sound speed model (dashed line), full waveform inversion results in the static model (circles), full waveform inversion results in the time-dependent model (crosses), and imaging by prestack depth migration (squares).

theoretical one above the wavenumber of $0.1 \mathrm{cpm}$ because of the regularization of the least squares solution. This produces a smoother sound speed model than the true model. Both the inversion with a $1 \mathrm{D}$ reference and constant reference model (see also supplemental material) recover the KolmogorovObukhov spectrum for the intermediate to low wavenumbers. For the reference, we also plot the energy spectrum estimated using the scaled reflectivity image. The imaging recovers a much shorter interval of the theoretical spectrum than FWI, and only near the high wavenumber limit.

\section{2) Model Resolution}

The spatial resolution of linearized waveform inversion depends on the frequency, source-receiver aperture, and background sound speed at the scattering point. Resolution can be characterized by the magnitude of the scattering wavenumber vector (Virieux and Operto 2009):

$$
\mathbf{k}_{\mathrm{SR}}=\frac{\omega}{\pi c_{0}} \cos \left(\frac{\theta}{2}\right) \mathbf{n},
$$

where $\theta$ is the scattering or aperture angle and $\mathbf{n}$ is a unit vector in the direction of $\mathbf{k}_{s}+\mathbf{k}_{r} ; \mathbf{k}_{s}, \mathbf{k}_{r}$ are propagation directions of rays from the source and receiver to the scattering point. The maximum wavenumbers (smallest features) are resolved for normal-incidence reflections 

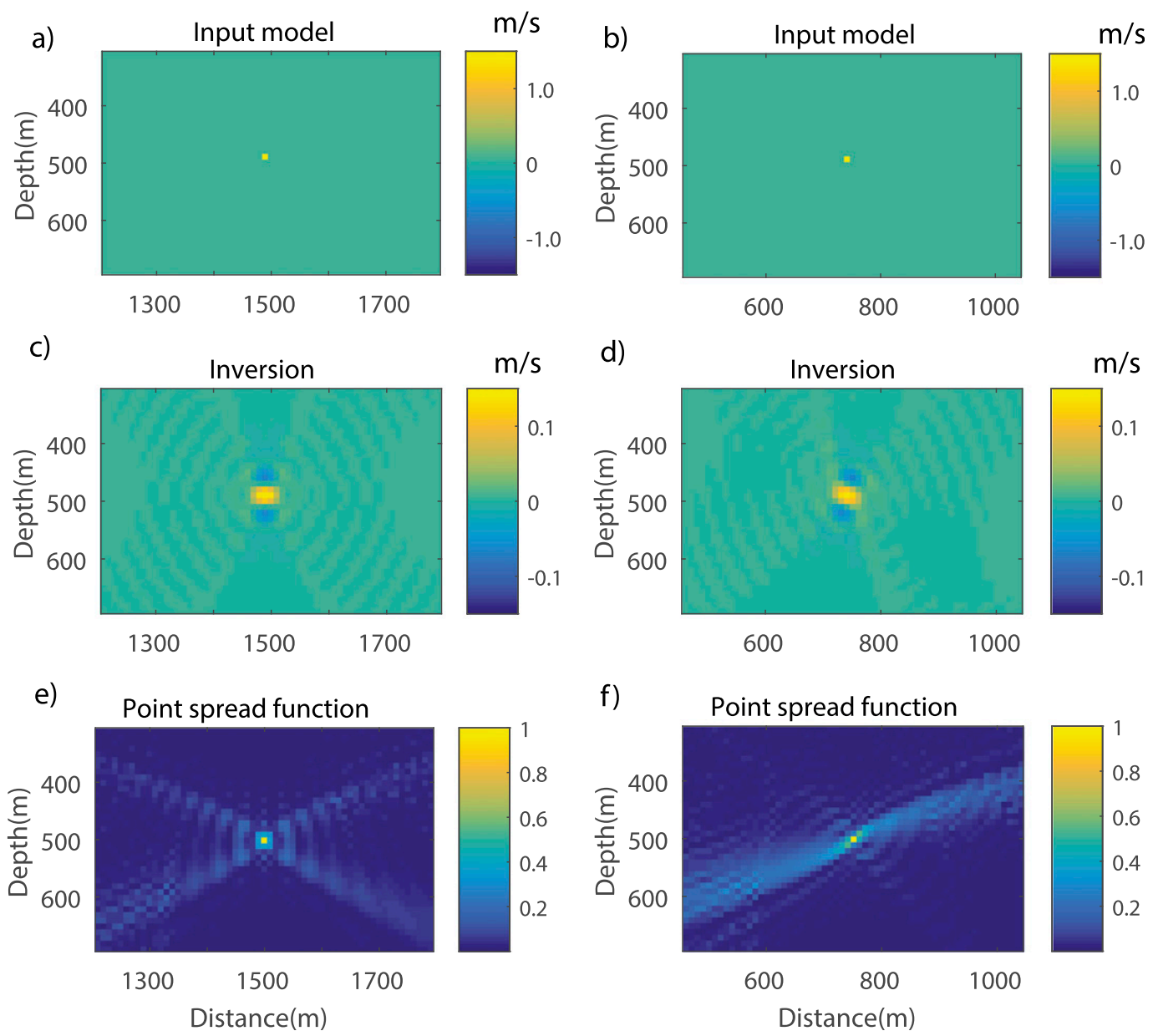

FIG. 7. Resolution tests. A recovery of the $10 \mathrm{~m} \times 10 \mathrm{~m}$ point perturbation of sound speed $\left(+1 \mathrm{~m} \mathrm{~s}^{-1}\right)$ by the waveform inversion from the (left) model center and (right) left side of the model. (a),(b) Input point perturbation; (c),(d) model recovery by inversion; and (e),(f) point spread function. The recovered magnitude of sound speed is reduced because of spreading of the input anomaly and development side lobes (in the vertical direction). The test shows a larger smearing and asymmetry of the reconstructed perturbation caused by poor ray coverage at the side of the model.

$(\theta=0)$. Therefore, in our applications it should be possible to resolve a sound speed perturbation of $37.5 \mathrm{~m}$ (half of the acoustic wavelength) using $20-\mathrm{Hz}$ acoustic waves in the inversion.

To assess the resolution in different regions of the model, we have performed several tests with a point perturbation. We invert a single point perturbation $(10 \mathrm{~m} \times 10 \mathrm{~m})$ of $+1 \mathrm{~m} \mathrm{~s}^{-1}$ at the depth of $500 \mathrm{~m}$ and the profile distance of 750 and $1500 \mathrm{~m}$ (Figs. 7a-d). In addition, we constructed a point spread function (Figs. 7e,f) that is based on an asymptotic representation of the Hessian matrix (Lecomte 2008). The point spread function can be expressed as

$$
\operatorname{PSF}\left(\mathbf{x}, \mathbf{x}^{\prime}, \omega\right)=\left|\sum_{s, r} \exp \left[i \omega \nabla T\left(\mathbf{x}, \mathbf{x}_{s}, \mathbf{x}_{r}\right) \cdot\left(\mathbf{x}^{\prime}-\mathbf{x}\right)\right]\right|,
$$

where $T\left(\mathbf{x}, \mathbf{x}_{s}, \mathbf{x}_{r}\right)=T\left(\mathbf{x}, \mathbf{x}_{s}\right)+T\left(\mathbf{x}, \mathbf{x}_{r}\right) ; \mathbf{x}^{\prime}$ is the location of the point perturbation. We estimated the Hessian at the frequency of $10 \mathrm{~Hz}$.

The recovered point perturbation (Figs. $7 \mathrm{c}, \mathrm{d}$ ) is characterized by side rings and side lobes (in the vertical direction). The size of the recovered sound speed anomaly is about $100 \mathrm{~m}$ in the vertical direction (including the upper and lower negative lobes) and about $25 \mathrm{~m}$ in the horizontal direction. The point spread is associated with the small amplitude of the recovered perturbation when compared to the input model. The recovered perturbation is symmetric in the center of the model (Fig. 7c) while it is inclined at the left side of model (Fig. 7d). This indicates a better spatial resolution in the center. The larger tradeoffs, associated with the sound speed structure in the vicinity of the imaging point 

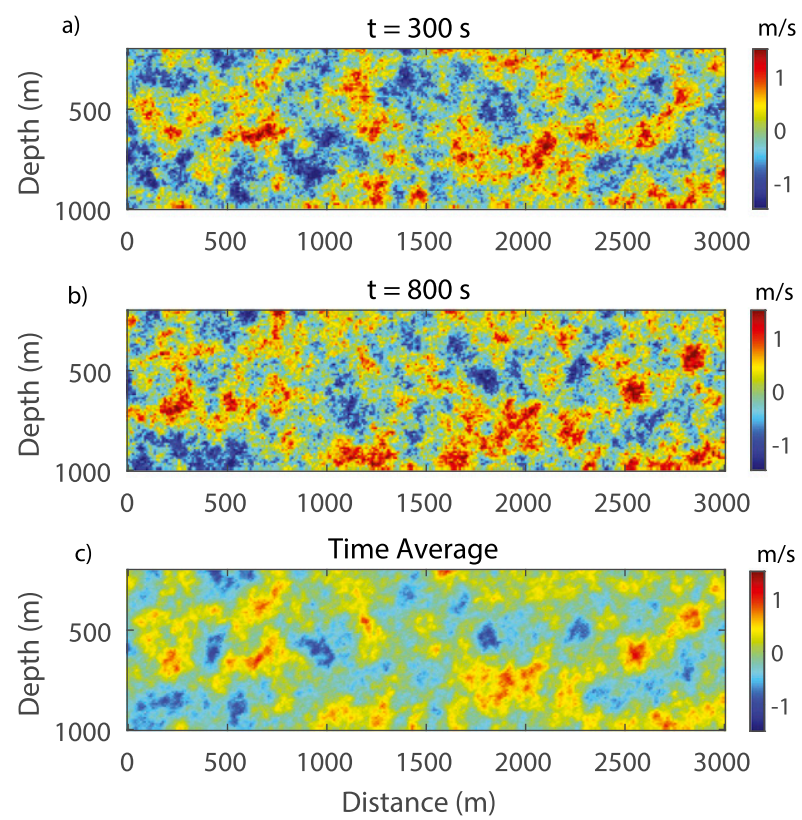

FIG. 8. Realizations of the turbulence model (sound speed field): elapsed time of (a) $300 \mathrm{~s}$ and (b) $800 \mathrm{~s}$, and (c) the model averaged over all time realizations (1500 s).

at the side of model in comparison with the model center, are also clear from Figs. 7e,f.

\section{3) NONSTATIONARY MODEL OF SOUND SPEED}

We proceed with application of our FWI algorithm to a time-dependent ocean model. First, we produce synthetic FD data in such a way that the sound speed model is modified at every shot time. The time evolution of the model is controlled by the time decorrelation function in Eq. (3). The ray-Born FWI setup includes the same parameters as in the static case. We assume that the 1D reference model is fixed.

Figures $8 \mathrm{a}$ and $8 \mathrm{~b}$ show random realizations of the turbulence model at 300 and $800 \mathrm{~s}$. The mean model over 120 realizations is shown in Fig. 8c. We observe that the realizations are not completely decorrelated, and the mean model is representative for the ensemble. The averaging over realizations acts as a low-pass filter applied to the model. The results of the waveform inversion [Eqs. (32) and (33)] and depth imaging [Eq. (35)] are presented in Figs. 9a and 9b. A conventional seismicoceanography image is obtained using normal move-out correction and stacking of common midpoint seismograms. The corresponding image of sound speed is presented in Fig. 9c.

The inversion process is stable and the misfit reduction proceeds to iteration 40 . At the higher frequencies $(>20 \mathrm{~Hz})$ the inversion starts diverging due to high nonlinearity. We halt iterations at this point. The
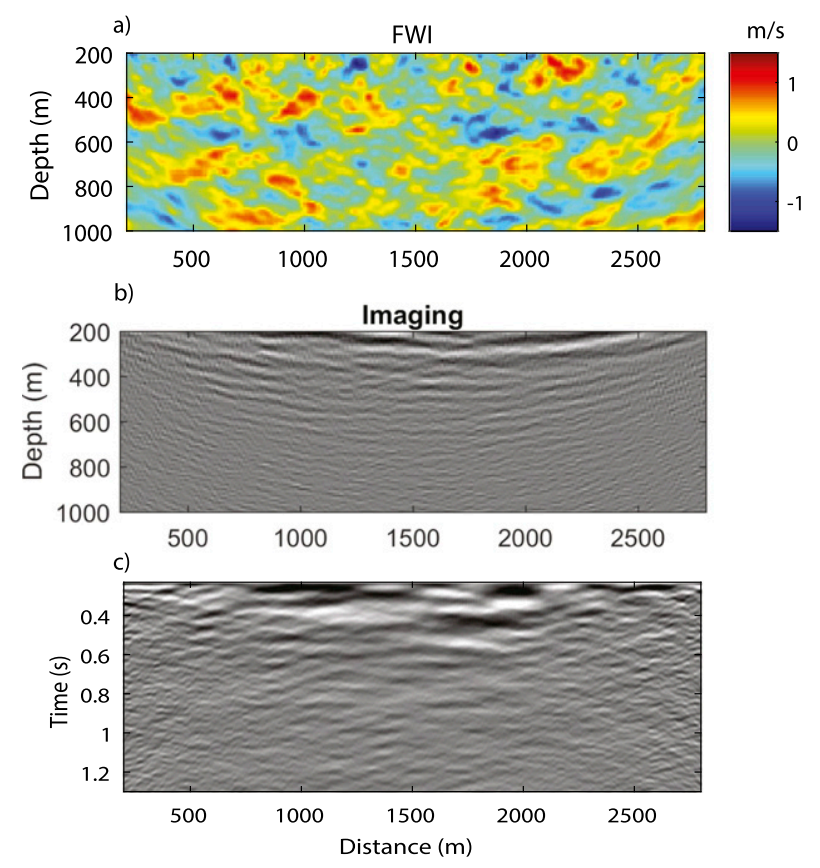

FIG. 9. Reconstruction in the time-dependent ocean model: (a) full waveform inversion results, (b) imaging using prestack depth migration, and (c) imaging by stacking common-mid-point seismograms corrected for normal move-out.

inverted model (Fig. 9a) contains a general pattern of the sound speed perturbation close to the time-averaged model (Fig. 8c). The imaging produces a more horizontally oriented pattern and has a problem to recover near-vertical or isometric structures (Figs. 9b,c). The energy spectrum (Fig. 6) computed using the inverted model shows that, even in this case, the KolmogorovObukhov slope is recovered well except for the high wavenumbers (similarly to the static case). The wavenumbers smaller than $0.07 \mathrm{cpm}$ (structures larger than $100 \mathrm{~m}$ ) are well recovered (in the statistical sense).

A nonstationary ocean model implies significant distortion of the waveforms (Fig. 3b) that is characterized by both phase shifts and amplitude changes. The efficient stable solution to this problem is provided by our multiscale nonlinear inversion approach. The convergence is achieved by extracting first the large-scale coherent characteristics of the medium and gradually introducing more details. This makes our approach specifically stable with respect to noise.

\section{4) OCEAN STRATIFICATION}

The structure of ocean turbulence is reported to be anisotropic in such a way that the vertical length scale is smaller than the horizontal length scale (e.g., Thorpe 2007). However, the degree and amount of anisotropy is not well constrained and would require additional 
complications in the stochastic modeling. Therefore, we leave the theoretical investigation of anisotropy for future studies. However, anisotropic effects have been shown to be important in the context of long-range sound propagation and acoustic tomography (Flatte and Colosi 2008). In particular, the horizontal correlation length of sound speed perturbations induced by internal waves can be one to two orders of magnitude larger than the vertical length.

The ocean stratified sound speed structure is addressed in our calculations by incorporating positive and negative slab-like sound speed anomalies superimposed on the isotropic turbulence structure (Fig. 10a). The slabs are characterized by sharp boundaries and a 2-3 times larger magnitude than the turbulence perturbations $\left( \pm 5 \mathrm{~m} \mathrm{~s}^{-1}\right)$ and thus dominate in the seismic signal. Despite these complications, the sound speed model is well reconstructed with our waveform inversion method (Fig. 10b). In Fig. 10c, the imaging by stacking common midpoint seismograms corrected for normal move-out is applied on the synthetic waveform data. The boundaries of stratified layers are well recovered while the turbulence structure is missing in the image. Thus, waveform inversion must be performed on real data in order to obtain information about diapycnal mixing in the ocean. Moreover, state-of-the-art acquisition instrumentation employed by commercial exploration seismics should provide sufficient data quality for further implementation of the proposed method on real data. Our test example is provided in the next section.

\section{Real data example}

The described FWI method has been applied to seismic oceanography data from the western Barents Sea continental margin, off Bear Island in the northeast Atlantic Ocean. A multichannel seismic reflection profile was acquired by the University of Bergen along a mainly west-east direction (close to $75^{\circ} \mathrm{N}$ ) in September 2006 (Libak et al. 2012, 2013), suborthogonal to the direction of the West Spitsbergen Current.

A 3000-m-long digital 240-channel seismic streamer was towed at $5 \mathrm{~m}$ depth while the recording hydrophone group length was $12.5 \mathrm{~m}$. The acoustic source consisted of a tuned air-gun array (five Bolt air guns) with a total volume of 1406 cubic inches and a peak frequency of around $10 \mathrm{~Hz}$. The shots were fired every $50 \mathrm{~m}$ at a depth of $6 \mathrm{~m}$. The data processing included removal of noisy traces, bandpass filtering, and $f-k$ filtering to remove the direct wave and coherent noise due to bubble pulses.

The main thermocline that separates warm, less dense Atlantic waters above from cold and denser waters below is marked by an increased reflectivity between 200
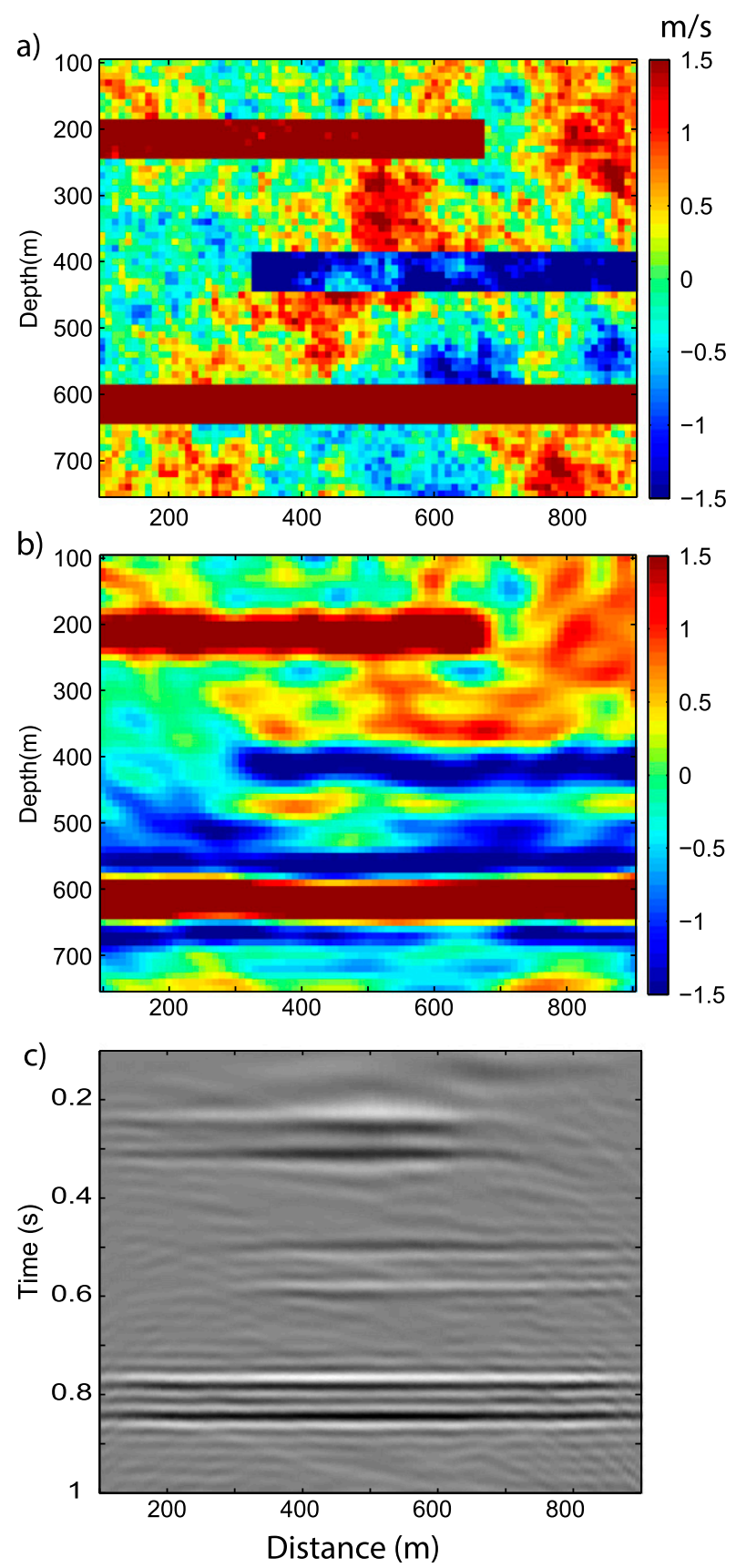

FIG. 10. Reconstructed model of sound speed containing turbulent structure superimposed on stratified perturbations. The magnitude of the slab anomalies is $\pm 5 \mathrm{~m} \mathrm{~s}^{-1}$. (a) The input model, (b) the full waveform inversion results, and (c) reconstruction done by stacking common midpoint seismograms corrected for normal move-out.

and $1000 \mathrm{~ms}$ in Fig. 11. The reflectivity pattern changes approaching the continental slope. The highly reflective zone becomes wider and more irregular. The upward concave reflectors crossing the ocean stratification can be interpreted as fronts of internal waves. We interpret 


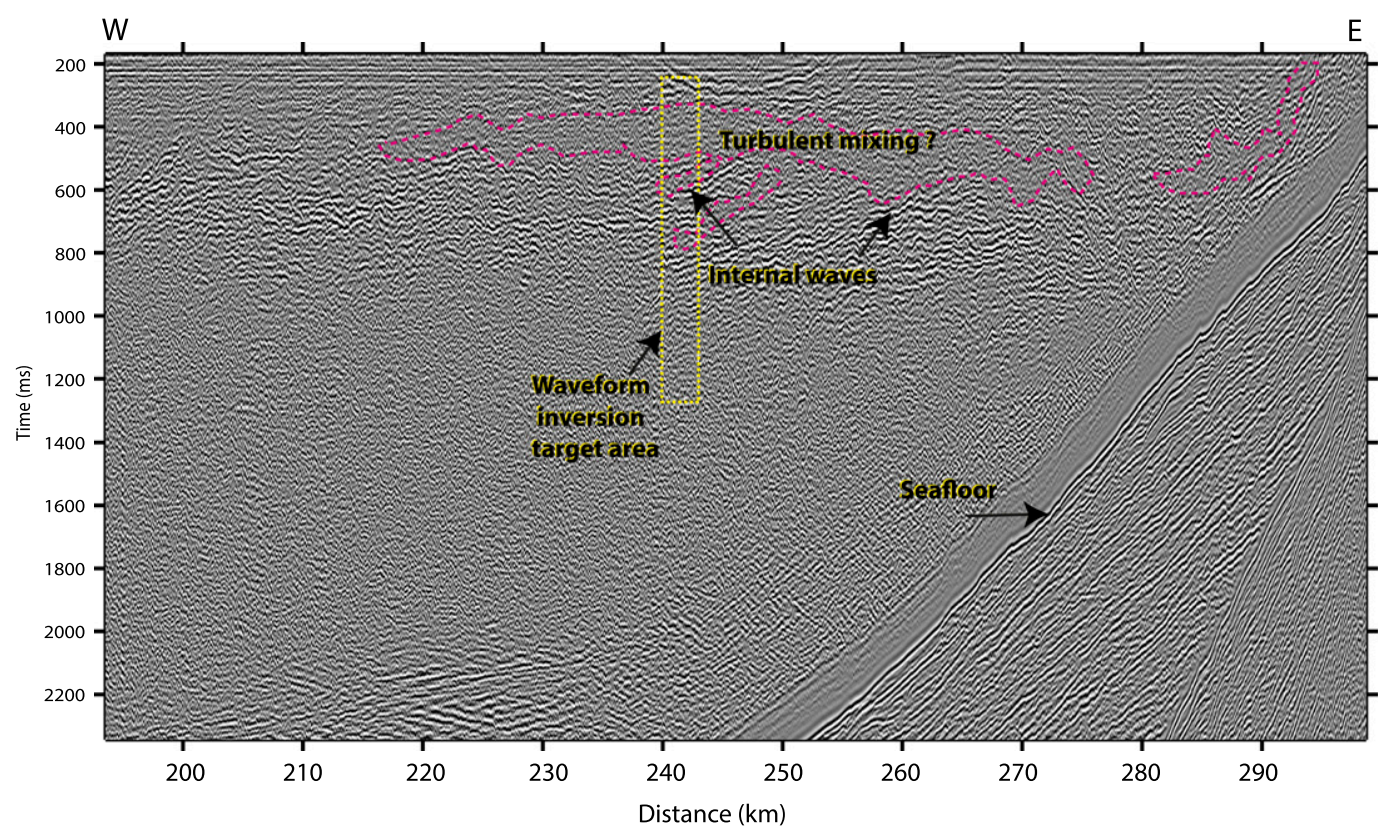

FIG. 11. The stacked multichannel reflection seismic data near the western Barents Sea continental slope in the northeast Atlantic Ocean (Libak et al. 2012, 2013).

the more transparent areas adjacent to the internal wave fronts as zones of intense turbulent mixing.

For the real data inversion, we used 3D Green functions [Eq. (21)]. However, the inversion was limited to a vertical $2 \mathrm{D}$ plane that contained the source and receiver locations. This ignores 3D side-scattering effects. However, these are likely to be small as the air-gun array that was employed is the one that is typically used in $2 \mathrm{D}$ seismic data acquisition and that emits most energy in the in-plane direction (i.e., the plane that contains the receivers) and much less to the sides.

We have performed the waveform inversion on the processed seismic data using 40 frequencies in the range of $3-15 \mathrm{~Hz}$ targeting the area that is presumably affected by turbulent flow (Fig. 11). We used a $10-\mathrm{Hz}$ Ricker wavelet as an acoustic source time function. The amplitudes of observed waveforms were scaled by the maximum amplitude obtained from dynamic ray tracing. The background temperature-sound speed structure was derived from CTD measurements performed along a nearby hydrographic transect in the same year (Walczowski 2014). The target area covers the depth interval of $200-1000 \mathrm{~m}$ corresponding to the main thermocline. The imaging of the upper $200 \mathrm{~m}$ of the ocean including the mixed layer would require an accurate separation of nearly horizontally scattered waves from the direct wave. In our application we assume a laterally homogeneous temperature and sound speed in the mixed layer.
We apply both the waveform inversion and imaging methods (discussed in the synthetic examples in section 5). They are shown to be complementary to invert seismic reflection data for the detailed sound speed structure. Taking into account that the scattered signal is very subtle and can be heavily affected by noise, it is important to compare inversion results based on different methods.

The sound speed perturbation obtained with respect to the 1D reference model using the FWI is shown in Fig. 12a. Subvertical upward concave structures can be observed below a reflector at about $250 \mathrm{~m}$. At the depth of $250-800 \mathrm{~m}$, isometric positive and negative sound speed anomalies with a diameter of $200-400 \mathrm{~m}$ are resolved. The coincident point measurements of the sound speed would be required to convert our sound speed perturbations to absolute values.

The contours of sound speed anomalies obtained using the FWI are consistent with our imaging results. The reflectors form at sharp gradients of the sound speed. The most prominent reflectors are located at about 250 , $400-500$, and $600 \mathrm{~m}$ depth (Figs. 12b,c). These are interpreted as internal wave fronts or large eddies (profile distance $240-255 \mathrm{~km}$ in Fig. 11). The turbulencedominated region is sandwiched between the reflectors at 250 and $600 \mathrm{~m}$ depth (Figs. 11, 12).

We estimated the power spectral density of the sound speed perturbation (in the $z$ direction) using an averaged periodogram (Fig. 13). The computed spectrum is 


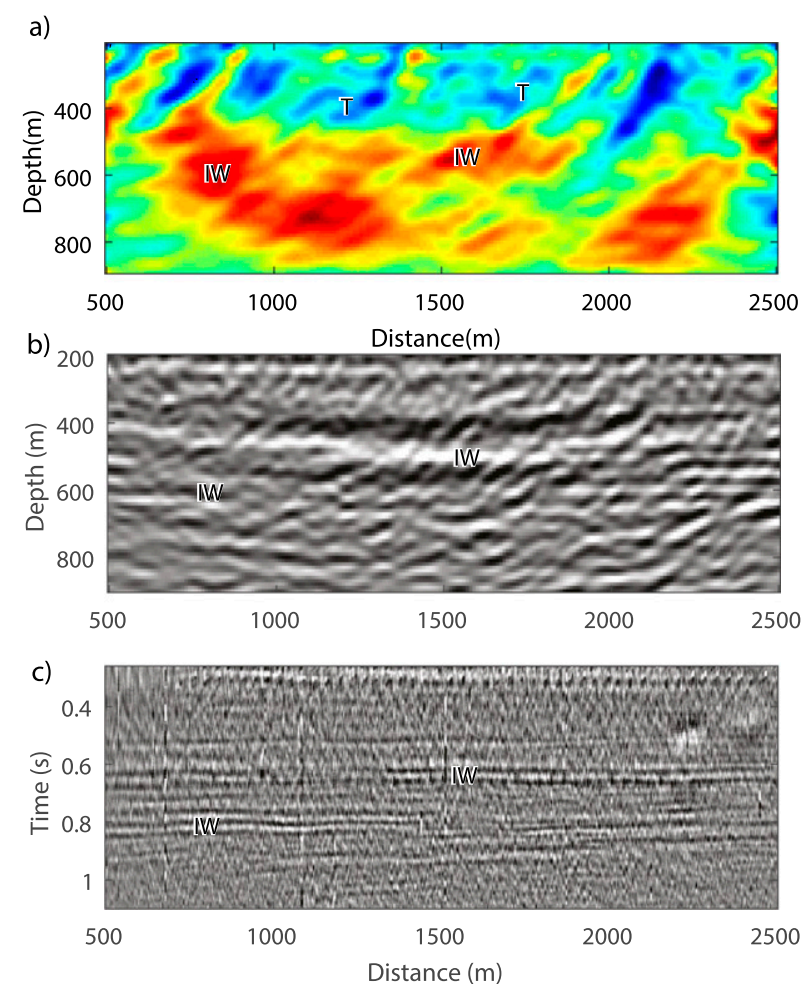

FIG. 12. A detailed model of the ocean sound speed based on real seismic oceanography data. The reference 1D sound speed profile is shown in Fig. 2. (a) Full waveform inversion results using normalized waveforms. The positive and negative sound speed anomalies correspond to the red and blue regions, respectively. The maximum absolute value based on ray-tracing amplitudes is about $1.5 \mathrm{~m} \mathrm{~s}^{-1}$. (b) Imaging using prestack depth migration. (c) Imaging by stacking common midpoint seismograms corrected for normal move-out. The "T" and "IW" labels denote the regions characterized by the presence of turbulence and internal waves, respectively.

compared with the theoretical turbulence spectrum. For comparison, we plot the power spectrum with the exponent -2 that approximates the empirical internal wave spectrum. The decay of spectral amplitudes with wavenumber follows the Kolmogorov-Obukhov law below $0.07 \mathrm{cpm}$ (Fig. 13), similarly to our synthetic results. This implies a maximum sound speed perturbation of about $1-2 \mathrm{~m} \mathrm{~s}^{-1}$. The spectral pattern indicates that the ocean flow in the study area is probably affected by both turbulence and internal waves. The next step toward more accurate inversion results should be the implementation of a more detailed background model of sound speed that would include internal waves and other larger-scale features. This, however, will require additional oceanographic data and/or acoustic travel-time tomography to constrain the sound speed since the large-scale features $(\geq 500 \mathrm{~m})$ would require very low frequencies $(\leq 3 \mathrm{~Hz})$ and/or a large

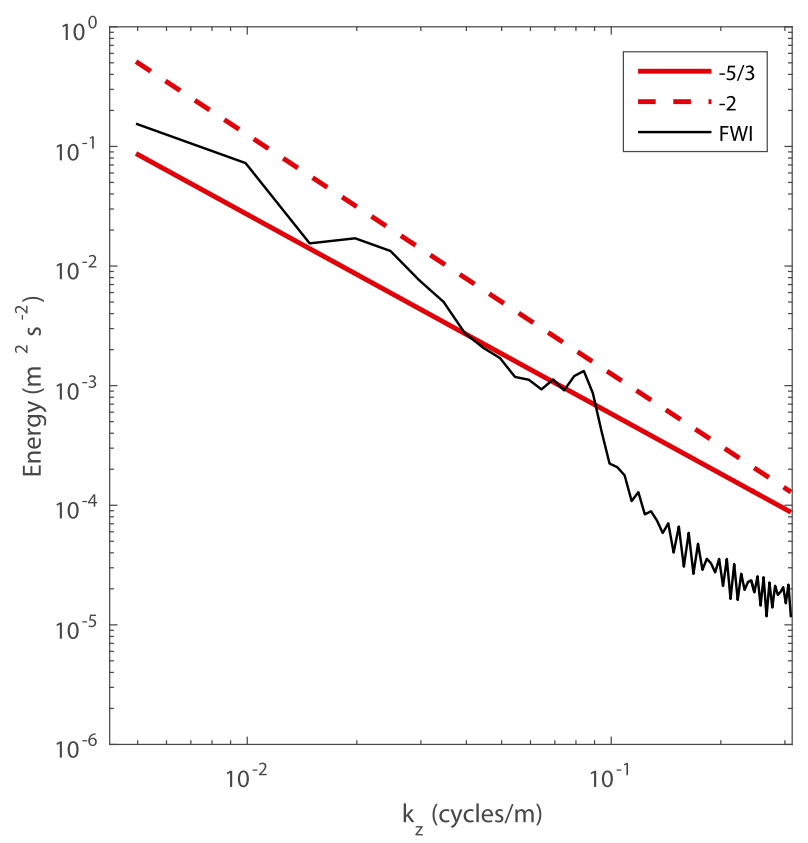

FIG. 13. The power spectral density estimated using the sound speed model obtained from the full waveform inversion applied to real seismic reflection data (black curve). The KolmogorovObukhov and internal wave spectra are shown by the red solid and dashed lines, respectively.

source-receiver aperture to be resolved by waveform tomography.

\section{Discussion and future perspectives}

The study and detection of turbulence is essential to understand the transport and dissipation of energy in the ocean. Here, we simulate the temperature field in an evolved turbulent ocean flow using a stochastic numerical model. Our ocean turbulence temperature model is developed based on the model of isotropic incompressible turbulent flow by Sabelfeld and Kurbanmuradov (1998). The random field simulation technique allows us to compute the time-dependent temperature field (Fig. 8) based on a spatial-temporal spectral tensor proposed in Kurbanmuradov (1997). The suggested randomization technique can accurately recover the mean over the ensemble. This makes the technique attractive to study the observations dealing with time-averaged information such as sampling the fluid heterogeneity with low-frequency sound.

Our numerical analysis addresses the limits of the single scattering assumption to study the ocean sound speed heterogeneity by comparison with a full numerical simulation (Fig. 3). We first compute random realization for the fully developed turbulent flow in the ocean. After that we compute theoretical seismograms using both an 
FD and an approximate ray-Born method. We confirm that a low-cost ray-Born scattering method is accurate enough to model and predict the isotropic turbulence structure at the source-receiver offsets $(1-3 \mathrm{~km})$ and propagation times $(0.2-1.5 \mathrm{~s})$ characteristic for seismic oceanography (Figs. 3, 5).

The energy spectrum estimated from the sound speed models both for $1 \mathrm{D}$ and a homogeneous reference model reproduces the energy spectrum of true model and the theoretical Kolmogorov-Obukhov spectrum for the wavenumbers $0.01-0.1 \mathrm{cpm}$ (Fig. 6). The higher wavenumbers are less well resolved because of limited model resolution and spatial smoothing. The estimation of the energy spectrum using imaging is only successful for intermediate wavenumbers. Thus, we conclude that, with respect to the spectral estimates, the FWI provides better statistical estimates of the ocean heterogeneity than imaging.

The imaging techniques based on migration of seismic reflection data recover spatial derivatives of the model parameters (Bleistein et al. 2013). In the frequency domain, the spectrum of the derivative can be expressed as $k^{2} E(k)$. The condition upon which the derivative exists is (Monin and Yaglom 1971)

$$
\int_{0}^{\infty} k^{2} E(k) d k<\infty
$$

The ocean flow is often described by a power-law function. In our case, $E(k) \sim k^{-5 / 3}$, which makes the integral in Eq. (38) diverge. This means that (in the least squares sense) the spatial gradient sought by imaging techniques cannot be defined. Thus, from the theoretical point of view, the FWI is a more appropriate method to recover the characteristics of the turbulent ocean flow than imaging. Our results show a limited recovery of the sound speed model using the imaging and thus support this theoretical conclusion.

In this paper, we considered the temperature random field described by the Kolmogorov-Obukhov spectrum. This certainly is a simplified model that cannot describe real large-scale flow. The experimental evaluation of the energy spectrum shows that the Kolmogorov-Obukhov power-law exponent $-5 / 3$ holds only in some part of the total range of wavenumber values. Nevertheless, we believe that the present study proves the accuracy and numerical efficiency of our method. Furthermore, the proposed stochastic modeling method to simulate the ocean temperature or sound speed fields can be extended to study more realistic flow models using other spectral representations. For example, Goodman (1990) considered an empirical anisotropic spectrum obtained from oceanographic measurements. This one-dimensional spectrum is represented by gluing together the functions of wavenumbers with different power-law exponents in the buoyancy-dominated, inertial, and Batchelor subranges, respectively. The experimental measurements of ocean temperature spectrum are presented, for example, in Gargett (1985) and Van Haren and Gostiaux (2009). Energy and temperature spectra of stably stratified turbulence were studied in Holloway (1986) and Kimura and Herring (2012).

For simplicity, we assumed that the acoustic scattering is mainly affected by temperature and less by salinity (density) variation. In the real ocean that is affected by complex interactions of various physical processes, these variations can be important (Sallarès et al. 2009). The Arctic-northeast Atlantic waters are known for the salinity inversion, such as a warmer and more saline water layer occurs in the top of the ocean stratification (Walczowski 2014). This situation is favorable for a double-diffusive small-scale convection.

The density variation related to salinity changes has a minimal impact on the sound speed. However, it may affect amplitudes of backscattered acoustic waves. This indicates that exploring the effects of density heterogeneities on the scattering amplitudes and waveform inversion is a natural way forward. In this case, it is straightforward to incorporate the corresponding density variations in the scattering integral Eq. (20) (Dahlen et al. 2000). In addition, with the ray-Born formulation of the forward problem, it is possible to tackle a multiparameter inversion through a Monte Carlo inversion approach such as in Cordua et al. (2012).

\section{Conclusions}

We develop a stochastic time-dependent model of the ocean turbulent temperature field based on the spectral model of isotropic incompressible flow. We use this model to produce 2D synthetic acoustic data similar to those employed in seismic exploration. We show that a migration technique commonly used in seismic oceanography may fail to recover a random field, characterized by a power-law function of wavenumbers, such as in the case of ocean turbulence. To avoid this limitation, we present a method for full seismic waveform inversion incorporating a ray-Born method and use it to predict temperature structure due to turbulence. We develop and apply the acoustic waveform inversion linearized with the Born approximation to study the ocean heterogeneity. The ray-Born scattering method was evaluated to yield sufficiently accurate forward modeling and full waveform inversion results at a low cost relative to the finite difference method when applied to seismic oceanography data. The presented inversion strategy accurately reproduces both the true model and the theoretical 
turbulence spectrum. We apply our waveform inversion method to real seismic data at the west Barents Sea continental margin in the northeast Atlantic. The obtained model shows sound speed variations that are probably related to a superposition of several diapycnal mixing processes in the vicinity of the continental slope. We conclude that the presented method offers a useful alternative to purely numerical waveform inversion methods and can be used to estimate both deterministic and statistical properties of the ocean heterogeneity.

Acknowledgments. We thank B. O. Ruud for providing results of data processing that were partly used in this paper. We also thank I. Fer for useful discussions and contribution at an early stage of this work. Comments by R. Hobbs and W. T. Wood on an earlier version of the paper were very useful. We thank the anonymous reviewers for constructive criticism and suggestions to improve the manuscript. A. Minakov acknowledges support from the Norwegian Academy of Science and Letters/ VISTA and the Research Council of Norway through its Centers of Excellence funding scheme, Project Number 223272. This research was partly done while H. Keers was on sabbatical at the University of Kiel and the University of Washington, and support for this by the L. Meltzer Høyskolefond, the Faculty of Mathematics and Natural Sciences of the University of Bergen, the Institute of Geoscience at the Christian Albrechts University of Kiel, the Applied Physics Laboratory at the University of Washington, and especially hosts Thomas Meier (CAU) and Bob Odom (UW), is gratefully acknowledged. D. Kolyukhin acknowledges financial support from the Russian Foundation for Basic Research (Grant 15-5520004). His study was partially supported by the Russian Academy of Sciences under the Arctic Programme.

\section{REFERENCES}

Aster, R. C., B. Borchers, and C. H. Thurber, 2011: Parameter Estimation and Inverse Problems. Academic Press, $376 \mathrm{pp}$.

Biescas, B., V. Sallars, J. L. Pelegr, F. Machn, R. Carbonell, G. Buffett, J. J. Daobeitia, and A. Calahorrano, 2008: Imaging meddy finestructure using multichannel seismic reflection data. Geophys. Res. Lett., 35, L11609, doi:10.1029/2008GL033971.

Bleistein, N., J. K. Cohen, and J. W. Stockwell Jr., 2013: Mathematics of Multidimensional Seismic Imaging, Migration, and Inversion. Interdisciplinary Applied Mathematics, Vol. 13, Springer, 510 pp.

Bornstein, G., B. Biescas, V. Sallarès, and J. Mojica, 2013: Direct temperature and salinity acoustic full waveform inversion. Geophys. Res. Lett., 40, 4344-4348, doi:10.1002/grl.50844.

Buffett, G. G., C. A. Hurich, E. A. Vsemirnova, R. W. Hobbs, V. Sallarès, R. Carbonell, D. Klaeschen, and B. Biescas, 2010: Stochastic heterogeneity mapping around a Mediterranean salt lens. Ocean Sci., 6, 423-429, doi:10.5194/os-6-423-2010.

Cerjan, C., D. Kosloff, R. Kosloff, and M. Reshef, 1985: A nonreflecting boundary condition for discrete acoustic and elastic wave equations. Geophysics, 50, 705-708, doi:10.1190/ 1.1441945 .

Červený, V., 2005: Seismic Ray Theory. Cambridge University Press, 722 pp.

Chernov, L. A., 1960: Wave Propagation in a Random Medium. McGraw-Hill, 168 pp.

Christakos, G., 2012: Random Field Models in Earth Sciences. Dover, $512 \mathrm{pp}$.

Claerbout, J. F., 1971: Toward a unified theory of reflector mapping. Geophysics, 36, 467-481, doi:10.1190/1.1440185.

Clayton, R. W., and R. H. Stolt, 1981: A Born-WKBJ inversion method for acoustic reflection data. Geophysics, 46, 15591567, doi:10.1190/1.1441162.

Coates, R., and C. Chapman, 1990: Ray perturbation theory and the Born approximation. Geophys. J. Int., 100, 379-392, doi:10.1111/j.1365-246X.1990.tb00692.x.

Colosi, J. A., and Coauthors, 1999: A review of recent results on ocean acoustic wave propagation in random media: Basin scales. IEEE J. Oceanic Eng., 24, 138-155, doi:10.1109/ 48.757267.

Cordua, K. S., T. M. Hansen, and K. Mosegaard, 2012: Monte Carlo full-waveform inversion of crosshole GPR data using multiple-point geostatistical a priori information. Geophysics, 77, H19-H31, doi:10.1190/geo2011-0170.1.

Dahlen, F., S.-H. Hung, and G. Nolet, 2000: Fréchet kernels for finite-frequency traveltimes-I. Theory. Geophys. J. Int., 141, 157-174, doi:10.1046/j.1365-246X.2000.00070.x.

DeSanto, J. A., 1992: Scalar Wave Theory. Springer Series on Wave Phenomena, Vol. 12, Springer, 193 pp.

Ewing, M., and J. L. Worzel, 1948: Long-range sound transmission. Geol. Soc. Amer. Mem., 27, 1-32, doi:10.1130/MEM27-3-p1.

Fichtner, A., 2011: Full Seismic Waveform Modelling and Inversion. Springer, $343 \mathrm{pp}$.

Flatte, S. M., and J. A. Colosi, 2008: Anisotropy of the wavefront distortion for acoustic pulse propagation through ocean sound-speed fluctuations: A ray perspective. IEEE J. Oceanic Eng., 33, 477-488, doi:10.1109/JOE.2008.2006341.

Gargett, A., 1985: Evolution of scalar spectra with the decay of turbulence in a stratified fluid. J. Fluid Mech., 159, 379-407, doi:10.1017/S0022112085003263.

Gonella, J., and D. Michon, 1988: Ondes internes profondes revelees par sismique reflexion au sein des masses d'eau en atlantique-est. C. R. Acad. Sci., Ser. II, 306 (12), 781-787.

Goodman, L., 1990: Acoustic scattering from ocean microstructure. J. Geophys. Res., 95, 11 557-11 573, doi:10.1029/ JC095iC07p11557.

Holbrook, W. S. and I. Fer, 2005: Ocean internal wave spectra inferred from seismic reflection transects. Geophys. Res. Lett., 32, L15604, doi:10.1029/2005GL023733.

—, P. Pramo, S. Pearse, and R. W. Schmitt, 2003: Thermohaline fine structure in an oceanographic front from seismic reflection profiling. Science, 301, 821-824, doi:10.1126/ science.1085116.

, I. Fer, R. W. Schmitt, D. Lizarralde, J. M. Klymak, L. C. Helfrich, and R. Kubichek, 2013: Estimating oceanic turbulence dissipation from seismic images. J. Atmos. Oceanic Technol., 30, 1767-1788, doi:10.1175/JTECH-D-12-00140.1.

Holliger, K., A. R. Levander, and J. A. Goff, 1993: Stochastic modeling of the reflective lower crust: Petrophysical and geological evidence from the Ivera Zone (northern Italy). J. Geophys. Res., 98, 11 967-11 980, doi:10.1029/93JB00351.

Holloway, G., 1986: Considerations on the theory of temperature spectra in stably stratified turbulence. J. Phys. Oceanogr., 
16, 2179-2183, doi:10.1175/1520-0485(1986)016<2179: COTTOT $>2.0 . \mathrm{CO} ; 2$.

Hudson, J., and J. Heritage, 1981: The use of the Born approximation in seismic scattering problems. Geophys. J. Int., 66, 221-240, doi:10.1111/j.1365-246X.1981.tb05954.x.

Ishimaru, A., 1999: Wave Propagation and Scattering in Random Media. John Wiley \& Sons, 600 pp.

Jensen, F. B., W. A. Kuperman, M. B. Porter, and H. Schmidt, 2011: Computational Ocean Acoustics. Springer, 794 pp.

Kimura, Y., and J. Herring, 2012: Energy spectra of stably stratified turbulence. J. Fluid Mech., 698, 19-50, doi:10.1017/ jfm.2011.546.

Klymak, J. M., and J. N. Moum, 2007: Oceanic isopycnal slope spectra. Part II: Turbulence. J. Phys. Oceanogr., 37, 12321245, doi:10.1175/JPO3074.1.

Kolyukhin, D., and K. Sabelfeld, 2005: Stochastic flow simulation in 3D porous media. Monte Carlo Methods Appl., 11, 15-37, doi:10.1515/1569396054027292.

Kormann, J., P. Cobo, M. Recuero, B. Biescas, and V. Sallarés, 2009: Modelling seismic oceanography experiments by using first-and second-order complex frequency shifted perfectly matched layers. Acta Acust. United Acust., 95, 1104-1111, doi:10.3813/AAA.918242.

_ - B. Biescas, N. Korta, J. de la Puente, and V. Sallarès, 2011: Application of acoustic full waveform inversion to retrieve high-resolution temperature and salinity profiles from synthetic seismic data. J. Geophys. Res., 116, C11039, doi:10.1029/ 2011JC007216.

Kraichnan, R. H., 1970: Diffusion by a random velocity field. Phys. Fluids, 13, 22-31, doi:10.1063/1.1692799.

Kramer, P. R., O. Kurbanmuradov, and K. Sabelfeld, 2007: Comparative analysis of multiscale Gaussian random field simulation algorithms. J. Comput. Phys., 226, 897-924, doi:10.1016/ j.jcp.2007.05.002.

Kurbanmuradov, O., 1997: Stochastic Lagrangian models for twoparticle relative dispersion in high-Reynolds number turbulence. Monte Carlo Methods Appl., 3, 37-52, doi:10.1515/ mcma.1997.3.1.37.

Lambaré, G., S. Operto, P. Podvin, and P. Thierry, 2003: 3D ray+ Born migration/inversion-Part 1: Theory. Geophysics, 68 , 1348-1356, doi:10.1190/1.1598128.

Lavery, A. C., R. W. Schmitt, and T. K. Stanton, 2003: Highfrequency acoustic scattering from turbulent oceanic microstructure: The importance of density fluctuations. J. Acoust. Soc. Amer., 114, 2685-2697, doi:10.1121/1.1614258.

Lecomte, I., 2008: Resolution and illumination analyses in PSDM: A ray-based approach. Leading Edge, 27, 650-663, doi:10.1190/ 1.2919584.

Libak, A., C. H. Eide, R. Mjelde, H. Keers, and E. R. Flüh, 2012: From pull-apart basins to ultraslow spreading: Results from the western Barents Sea margin. Tectonophysics, 514-517, 4461, doi:10.1016/j.tecto.2011.09.020.

—, P. Poor Moghaddam, A. Minakov, B. O. Ruud, H. Keers, and R. Mjelde, 2013: Seismic waveform inversion and imaging of deepwater glacial sedimentary fans in the northern Norwegian-Greenland Sea. Geophysical Research Abstracts, Vol. 15, Abstract EGU2013-6083. [Available online at http:// meetingorganizer.copernicus.org/EGU2013/EGU2013-6083.pdf.]

Mackenzie, K. V., 1981: Nine-term equation for sound speed in the oceans. J. Acoust. Soc. Amer., 70, 807-812, doi:10.1121/ 1.386920

Monin, A., and A. Yaglom, 1971: Statistical Fluid Mechanics: Mechanics of Turbulence. Vol. 2, MIT Press, $896 \mathrm{pp}$.
Moser, T. J., 2012: Review of ray-Born forward modeling for migration and diffraction analysis. Stud. Geophys. Geod., 56, 411-432, doi:10.1007/s11200-011-9046-0.

Operto, S., G. Lambare, P. Podvin, and P. Thierry, 2003: 3D ray+ Born migration/inversion-Part 2: Application to the SEG/ EAGE overthrust experiment. Geophysics, 68, 1357-1370, doi:10.1190/1.1598129.

Ostashev, V. E., and D. K. Wilson, 2015: Acoustics in Moving Inhomogeneous Media. 2nd ed., CRC Press, $541 \mathrm{pp}$.

Paige, C. C., and M. A. Saunders, 1982: LSQR: An algorithm for sparse linear equations and sparse least squares. ACM Trans. Math. Software, 8, 43-71, doi:10.1145/355984.355989.

Pratt, R. G., C. Shin, and G. Hick, 1998: Gauss-Newton and full Newton methods in frequency-space seismic waveform inversion. Geophys. J. Int., 133, 341-362, doi:10.1046/ j.1365-246X.1998.00498.x.

Ross, T., and R. Lueck, 2003: Sound scattering from oceanic turbulence. Geophys. Res. Lett., 30, 1343, doi:10.1029/ 2002GL016733.

Rytov, S., Y. Kravtsov, and V. Tatarskii, 1989: Principles of Statistical Radiophysics 4: Wave Propagation through Random Media. Springer, $188 \mathrm{pp}$.

Sabelfeld, K., 1991: Monte Carlo Methods in Boundary Value Problems. Springer, 283 pp.

_ 2010: Stochastic simulation for solving random boundary value problems and some applications. Large-Scale Scientific Computing, Springer, 26-39.

_ , and O. Kurbanmuradov, 1998: Two-particle stochastic EulerianLagrangian models of turbulent dispersion. Math. Comput. Simul., 47, 429-440, doi:10.1016/S0378-4754(98)00124-4.

Sagaut, P., 2006: Large Eddy Simulation for Incompressible Flows: An Introduction. Springer, $558 \mathrm{pp}$.

Sallarès, V., B. Biescas, G. Buffett, R. Carbonell, J. J. Dañobeitia, and J. L. Pelegrí, 2009: Relative contribution of temperature and salinity to ocean acoustic reflectivity. Geophys. Res. Lett., 36, L00D06, doi:10.1029/2009GL040187.

Sato, H., M. C. Fehler, and T. Maeda, 2012: Seismic Wave Propagation and Scattering in the Heterogeneous Earth. 2nd ed., Springer, $496 \mathrm{pp}$.

Seim, H. E., 1995: Sound scattering from oceanic turbulence. J. Atmos. Oceanic Technol., 12, 367-380, doi:10.1175/ 1520-0426(1995)012<0367:ABFTM>2.0.CO;2.

Symes, W. W., 2008: Migration velocity analysis and waveform inversion. Geophys. Prospect., 56, 765-790, doi:10.1111/ j.1365-2478.2008.00698.x.

Tarantola, A., 1984: Inversion of seismic reflection data in the acoustic approximation. Geophysics, 49, 1259-1266, doi:10.1190/1.1441754.

Tatarskii, V. I., 1961: Wave Propagation in Turbulent Medium, McGraw-Hill, 285 pp.

Tengesdal, H., A. Minakov, and H. Keers, 2014: Hybrid ray-Born and finite-difference full waveform inversion. 76th EAGE Conf. and Exhibition, Amsterdam, Netherlands, European Association of Geoscientists and Engineers, doi:10.3997/ 2214-4609.20140854.

Thierry, P., S. Operto, and G. Lambaré, 1999: Fast 2-d ray+ Born migration/inversion in complex media. Geophysics, 64, 162181, doi:10.1190/1.1444513.

Thorpe, S. A., 2007: An Introduction to Ocean Turbulence. Cambridge University Press, $240 \mathrm{pp}$.

— temperature microstructure. Limnol. Oceanogr., 28, 601-613, doi:10.4319/lo.1983.28.4.0601. 
Van Haren, H., and L. Gostiaux, 2009: High-resolution open-ocean temperature spectra. J. Geophys. Res., 114, C05005, doi:10.1029/ 2008JC004967.

Virieux, J., and S. Operto, 2009: An overview of full-waveform inversion in exploration geophysics. Geophysics, 74, WCC1-WCC26, doi:10.1190/1.3238367.

Vsemirnova, E., R. Hobbs, N. Serra, D. Klaeschen, and E. Quentel, 2009: Estimating internal wave spectra using constrained models of the dynamic ocean. Geophys. Res. Lett., 36, L00D07, doi:10.1029/2009GL039598.

Walczowski, W., 2014: Atlantic Water in the Nordic Seas. Springer, $174 \mathrm{pp}$.

Wood, W. T., W. S. Holbrook, M. K. Sen, and P. L. Stoffa, 2008: Full waveform inversion of reflection seismic data for ocean temperature profiles. Geophys. Res. Lett., 35, L04608, doi:10.1029/ 2007GL032359. 